Advanced Magnet Laboratory, November 2003

DOE SBIR Phase I Final Report: DE-FG02-02ER83359

\title{
SUPERCONDUCTING QUADRUPOLE ARRAYS FOR MULTIPLE BEAM TRANSPORT Project Summary
}

The goal of this research was to develop concepts for affordable, fully functional arrays of superconducting quadrupoles for multi-beam transport and focusing in heavy ion fusion (HIF) accelerators. Previous studies by the Virtual National Laboratory (VNL) collaboration have shown that the multi-beam transport system (consisting of alternating gradient quadrupole magnets, a beam vacuum system, and the beam monitor and control system) will likely be one of the most expensive and critical parts of such an accelerator. This statement is true for near-term fusion research accelerators as well as accelerators for the ultimate goal of power production via inertial fusion. For this reason, research on superconducting quadrupole arrays is both timely and important for the inertial fusion energy (IFE) research program. This research will also benefit near-term heavy ion fusion facilities such as the Integrated Research Experiment (IRE) and/or the Integrated Beam Experiment (IBX).

We considered a 2-prong approach that addresses the needs of both the nearer and longer term requirements of the inertial fusion program. First, we studied the flat coil quadrupole design that was developed by LLNL; this magnet is $150 \mathrm{~mm}$ long with a $50 \mathrm{~mm}$ aperture and thus is suitable for near term experiments that require magnets of a small length to aperture ratio. Secondly, we studied the novel double-helix quadrupole (DHQ) design in a small (3 $\mathrm{x} 3$ ) array configuration; this design can provide an important step to the longer term solution of low-cost, easy to manufacture array constructions. Our Phase I studies were performed using the AMPERES magnetostatic analysis software. Consideration of these results led to plans for future magnet $R \& D$ construction projects.

The first objective of Phase I was to develop the concept of a superconducting focusing array that meets the specific requirements of a heavy ion fusion accelerator. Detailed parameter studies for such quadrupole arrays were performed. Based on these studies, the primary magnet parameters and the general features required for a complete array system (including vacuum and cryostat) were identified. Basic system concepts were formulated to serve as guides for future development work.

A related issue was to compare the applicability and benefits of two different magnet technologies for use in such a quadrupole array. Analytical studies were performed for each of the two coil designs, a flat coil based on an HCX quadrupole designed by LLNL and a doublehelix quadrupole designed by AML. These studies have confirmed the feasibility of using either of the two coil designs in a small array. 
Advanced Magnet Laboratory, June 2003

DOE SBIR Phase I Final Report: DE-FG02-02ER83359

SUPERCONDUCTING QUADRUPOLE ARRAYS FOR MULTIPLE BEAM TRANSPORT

DOE SBIR Phase I Final Report

Table of Contents

Section

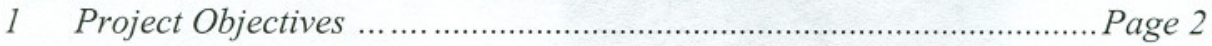

2 Phase I Results ..........................................................................Page 2

2.1 Parameter Studies and Beam Physics Issues ……………………......Page 2

2.2 Flat Coil Analysis............................................................................Page 3

2.3 Double-Helix Quadrupole (DHQ) Analysis …………………...........Page 6

2.4 Edge Termination Scheme....................................................................Page 8

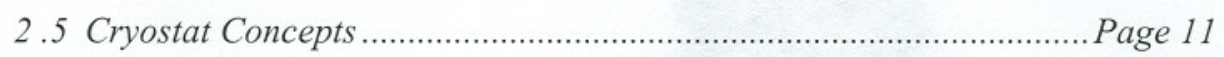

3 Technical Feasibility/ Future Research ………………………........ Page 13

3.1 Edge Termination for Flat Coils ......................................................Page 12

3.2 Double-Helix Quadrupole Array ...................................................Page 12

3.3 Cryostat Design and Construction................................................... 13

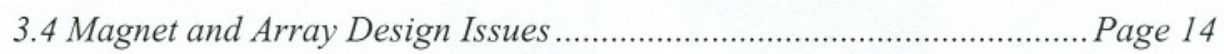

3.5 Accelerator System Issues.............................................................. Page 15 


\section{SUPERCONDUCTING QUADRUPOLE ARRAYS FOR MULTIPLE BEAM TRANSPORT}

\section{Project ObJectives}

The first objective of the Phase I research was to determine the specifications of the array, e.g., the aperture, length, and gradient of the individual quadrupoles and the number of beams in the array. This task proved to be far more difficult than originally anticipated, but completing the task led to a major unanticipated benefit, namely, the development of a new computer code MAG3D that integrates beam dynamics, magnetic field calculations with boundary conditions appropriate for quadrupole arrays, and important aspects of mechanical engineering. As the name indicates, the code calculates 3-dimensional fields. It includes optimizing routines that adjust the important variables such as aperture, length, and field to optimize a selected figure of merit -- subject to essential constraints on ion beam stability and mechanical feasibility. In this sense the code is a major first step toward a detailed accelerator optimization and design code.

The second objective was to perform analytical studies on the array performance of 2 different quadrupole designs. One array configuration used a cell with its four sides composed of flat coils based on an HCX quadrupole design by LLNL. The second configuration used array cells consisting of one double-helix quadruple (DHQ), a novel design that achieves a quadrupole field by the sinusoidal modulation of the axial position of the conductor turns in pairs of solenoid type coils.

\section{Phase I Results}

\subsection{Parameter Studies and Beam Physics Issues}

For our Phase I studies we chose average beam current density (beam current in a single beam divided by transverse area of the quadrupole containing the beam) as the principal figure of merit. Maximizing this figure of merit is an important consideration in designing low cost, high efficiency accelerators. Figure 1 shows typical results. It plots average beam current density as a function of beam tube radius. At each point on the curve, MAG3D varies a number of design parameters (e.g., amount of superconductor, quadrupole length, and magnetic field) to maximize the average beam current density. The optimal beam tube aperture is approximately $40 \mathrm{~mm}$, a value that we have chosen as our base case. The optimal length depends on the ion mass and kinetic energy, but the optimal aperture depends only weakly on these quantities. For our studies, we usually assumed a $16 \mathrm{MeV}$, singly charged potassium beam. This choice leads to an optimal quadrupole length of approximately $0.3 \mathrm{~m}-$ a convenient length to fabricate and test.

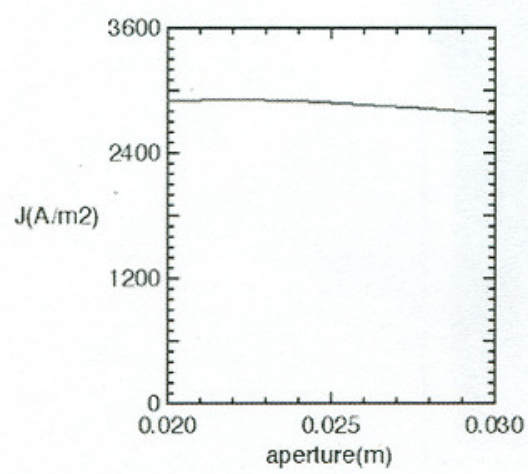

Figure 1. Average beam current density as a function of beam tube aperture radius. 
It is noteworthy that the optimal magnetic fields are rather modest. The peak fields at the superconductor are usually in the range of 3 to $5 \mathrm{~T}$. Higher fields actually lead to lower average beam current density because the increased size of the conductors and structures to support the conductors leads to a situation where the transverse area of the quadrupole increases more rapidly than the beam current.

The curve of average beam current density as a function of beam tube radius is based on standard niobium-titanium superconductor. Our studies indicate that improvements in superconducting cable would lead to higher beam current density and higher optimal fields but the improvement is modest. A $70 \%$ increase in the superconductor current density gives less than $10 \%$ improvement in average beam current density.

Finally, we note that the optimal parameters of a magnet depend strongly on the assumptions that one makes about beam alignment. For example, the optimal average beam current density increases by approximately $50 \%$ as the precision of beam alignment is improved from $5 \mathrm{~mm}$ to $0.5 \mathrm{~mm}$.

\subsection{Flat Coil Analysis}

The flat coil configuration was based on the LLNLr6 design that has been developed by Lawrence Livermore National Laboratory as a single aperture quadrupole for a near term HCX experiment. This configuration was first analyzed as a 2-layer coil with an iron yoke to establish the base line performance in this configuration and also compare it with previous calculations that were made at LLNL.[1] Figure 2 shows a view of the magnetic model that was used in the AMPERES 6.0 CAE software package for 3D simulation of magnetostatic systems. The results of this analysis were in good agreement with the previous work.

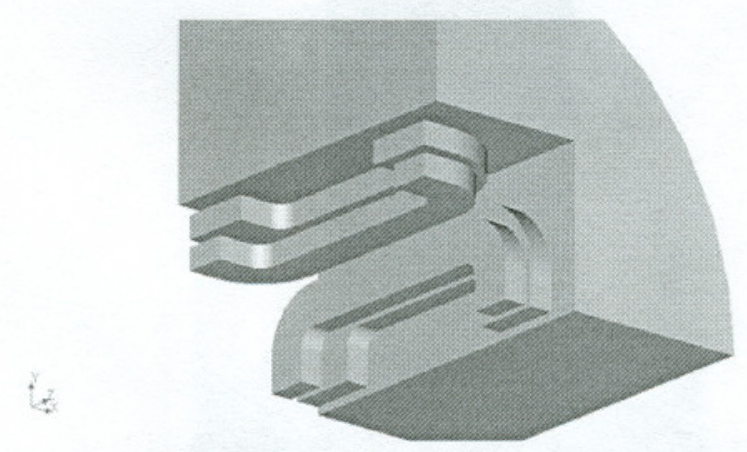

Figure 2. Magnetic model of LLNL6r2 design used to compute fields with AMPERES 6.0.

The next step was to investigate the use of this design as the basic element in an array. In this case the inner coil geometry was selected and placed in a $3 \times 3$ array. The magnetic model is shown in Figure 3. This model enabled a determination of the magnetic field characteristics for such an assembly, including the field enhancement in the central element due to the neighboring cells.

It is noted here that there was no attempt to apply edge termination to this model since that was beyond the scope of the Phase I project. When these studies were done, the edge termination scheme had not been developed to the point where it could be applied to such a model. 


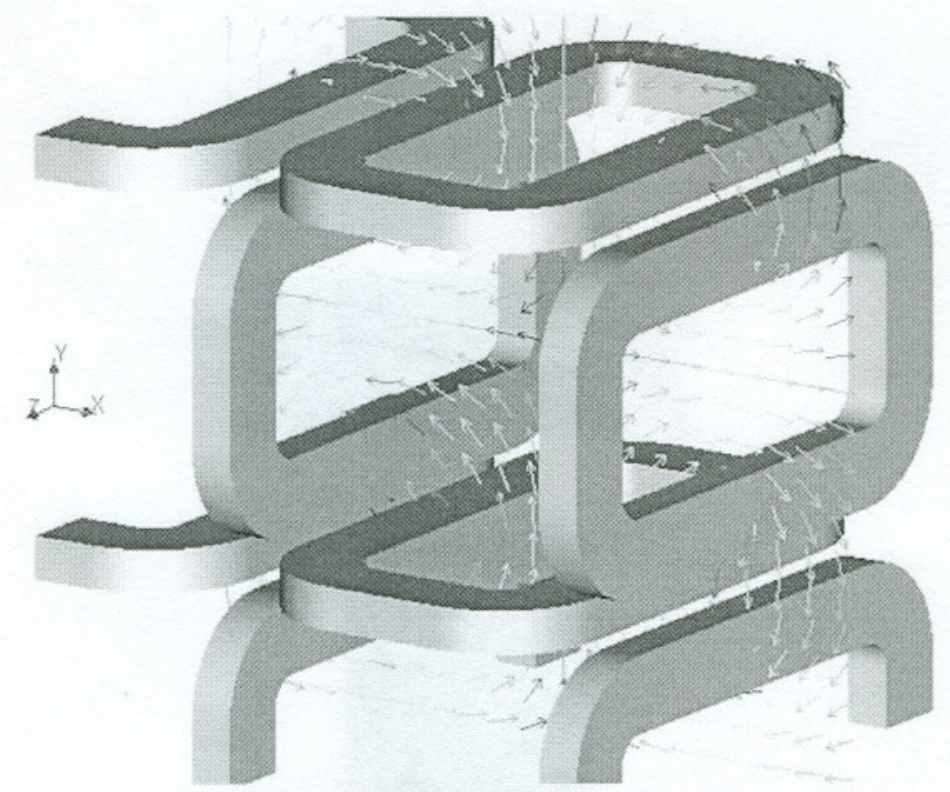

Figure 3. One-quarter model (with symmetry) used for magnetic field calculations in a $3 \times 3$ array composed of LLNL6r2 inner coils.

It is of interest to note some of the results of this analysis. For example, the fields in each of the representative type cells in the model in Figure 3 are shown in Figure 4. Since the flat coil design has a compact end that is mostly transverse to the axis, there is a pronounced axial field component in the ends of each cell, as shown.

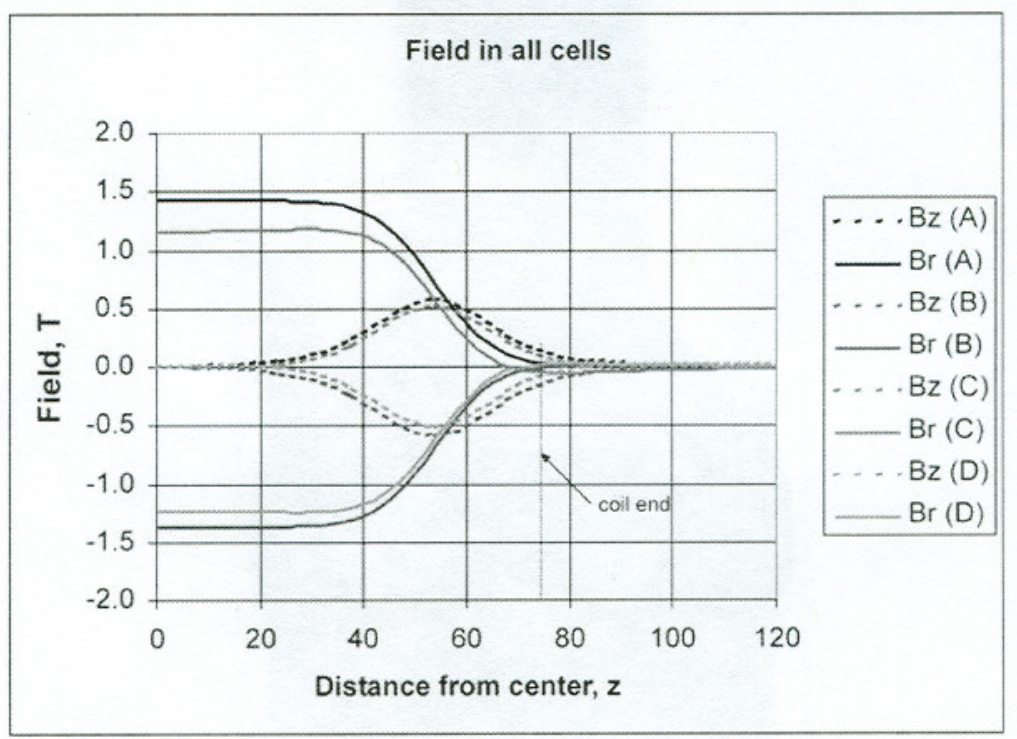

Figure 4. Variation of radial field $(\mathrm{Br})$ and axial field $(\mathrm{Bz})$ in the $3 \times 3$ array model at a reference radius of 25 $\mathrm{mm}$. Cell A is the central cell, cell B is the mid side cell, cell $C$ is the corner cell, and cell $D$ is the mid top cell. 
Table 1 compares the fields in array cells to a single cell without neighbors. It is seen that the field enhancement due to the array structure is about $26 \%$. The asymmetry of the fields in the neighboring cells is indicated by the offset of the maximum and minimum values of the field.

Table 1. Amplitude of field at $r=25 \mathrm{~mm}$ in different cells of the $3 \times 3$ array compared with a single cell. Cell $\mathrm{A}$ is the central cell, cell B is the mid side cell, cell $C$ is the corner cell, and cell $D$ is the mid top cell.

\begin{tabular}{|l|r|r|r|r|r|r|}
\hline Cell & \multicolumn{1}{c|}{ A } & \multicolumn{1}{c|}{ B } & \multicolumn{1}{c|}{ C } & \multicolumn{1}{c|}{ D } & \multicolumn{1}{c|}{ Single } & \multicolumn{1}{c|}{ C: Bt } \\
\hline Maximum B & 1.4270 & 1.3641 & 1.3863 & 1.4466 & 1.1332 & 1.3150 \\
\hline Minimum B & -1.4270 & -1.4466 & -1.3863 & -1.3641 & -1.1332 & -1.4789 \\
\hline Shift & 0.0000 & -0.0826 & 0.0000 & 0.0826 & 0.0000 & -0.1639 \\
\hline Gradient (T/m) & 57.08 & 56.21 & 55.45 & 56.21 & 45.33 & \\
\cline { 1 - 4 } Enhancement & $25.92 \%$ & $24.02 \%$ & $22.33 \%$ & $24.02 \%$ & $0.00 \%$ & \\
\cline { 1 - 4 } & & & & & &
\end{tabular}

For practical array applications, it is also of interest to study the force distribution between the various elements in the array. This was done for the model shown in Figure 3. The basic result was that the interior cells are in force equilibrium with their neighbors. However, the external array elements that are missing a coplanar neighbor on one side require the application of external reaction forces to compensate for the missing neighbor coil and keep the cells in equilibrium. This is illustrated in the diagram shown in Figure 5.

i:

Figure 5. Forces acting on the elements in the sample array. The flat coils of interior cells are in force equilibrium but coils that lack a coplanar neighbor on one side must be maintained in force equilibrium by the reaction forces, $R$, as indicated.

The magnitude of these external forces depends on the location of the flat coil. Thus, in this model the two exterior edge coils, labeled $\mathrm{H}$ and $\mathrm{J}$, require the same reaction force, and the two 
interior coils, labeled D and I, require the same reaction force. The corresponding equilibrium forces have the values shown in Table 2 .

Table 2. External reaction forces, $\mathrm{N} / \mathrm{mm}^{2}$, necessary to keep cells in equilibrium.

\begin{tabular}{|c|c|c|}
\hline Coils & $\mathrm{R}_{1}$ & $\mathrm{R}_{2}$ \\
\hline $\mathrm{D}, \mathrm{I}$ & 97.6 & \\
\hline $\mathrm{H}, \mathrm{J}$ & & 66.2 \\
\hline
\end{tabular}

\subsection{Double-Helix Quadrupole (DHQ) Analysis}

The double-helix quadruple (DHQ) whose geometry is shown in Figure 6 has been described in Reference [2]. This magnet achieves a quadrupole field by the sinusoidal modulation of the axial position, $z$, of the turns in pairs of solenoid type coils according to the relation $\mathrm{z}(\theta)=\frac{\mathrm{h} \theta}{2 \pi}+\mathrm{A}_{\mathrm{n}} \sin \mathrm{n} \theta$ where $h$ is the helical advance per turn, $A_{n}$ is the amplitude of the modulation and $n$ is the modulation frequency ( $n=2$ for the quadrupole.)

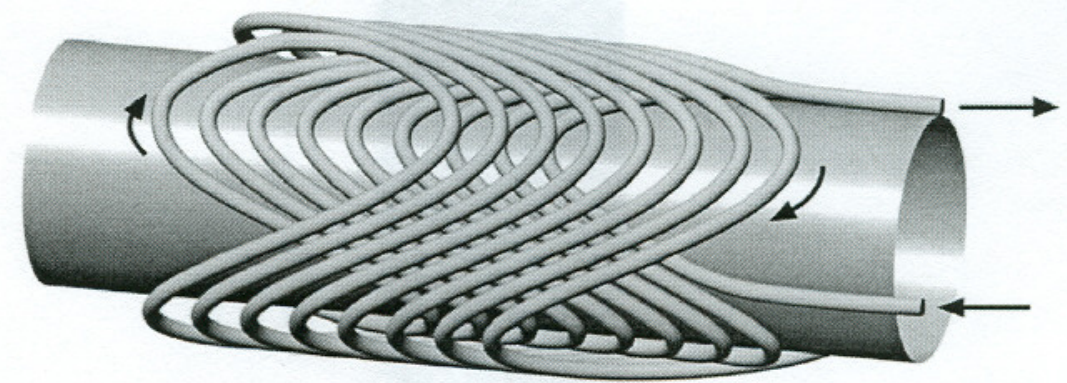

Figure 6. Example of the coil geometry used in the double-helix quadrupole (DHQ) for a 2-layer coil. The current directions shown cancel the solenoid field and add the quadrupole field from each layer.

This magnet appears to be a practical solution to the array quadrupole because it avoids many of the cost-driving problems associated with the flat coil and cosine $2 \theta$ coil configurations. Among the most important features for array applications are

- Elimination of splices between the four coil segments required in the conventional flat coil quadruple design; this reduces the resistive heat load due to the splice resistance, (typically $10^{-9} \Omega$ per splice).

- Simplified method of manufacture that uses conventional machine tools.

- Low cost coil parts that consist only of superconductor, composite tubes, epoxy for coil impregnation, and (if required) aluminum alloy cylindrical coil reinforcement.

- Virtually no error fields (12-pole and 20-pole harmonics) produced in the 2dimensional cross section.

It is recognized that this configuration may not be as adaptable to applications requiring short, large aperture coils since the ends are not as compact as in the flat coil design. The DHQ produces a more gradual drop in quadrupole field as the ends of the magnet are approached. This effect can be seen in Figure 7 that plots the quadrupole field strength at a radius of $16 \mathrm{~mm}$ in an 
example flat coil and DHQ coil of the same aperture; in this case both coils had an aperture between the conductors of $48 \mathrm{~mm}$.

In this plot, the DHQ field shows a more gradual drop off at the coil ends than the flat coil design. The effective drop off length of the DHQ can be adjusted somewhat by changing the winding modulation factor $\mathrm{k}$, where $\mathrm{k}=\frac{\mathrm{A}_{2}}{\mathrm{a}}$ with $\mathrm{A}_{2}$ (the amplitude of the modulation) and a (the radius of the coil). The plots of $\mathrm{Bt}(\mathrm{DHQ})$ and $\mathrm{Bz}(\mathrm{DHQ})$ in the figure are based on $\mathrm{k}=1$. The effect of using $\mathrm{k}=0.7$ is shown in the plot labeled as $\mathrm{Bt}(\mathrm{k}=.7)$ and we see that the field strength is reduced when $\mathrm{k}$ is decreased. It is important to note that the DHQ does not produce the axial field component in the ends that occurs in the flat coil design.

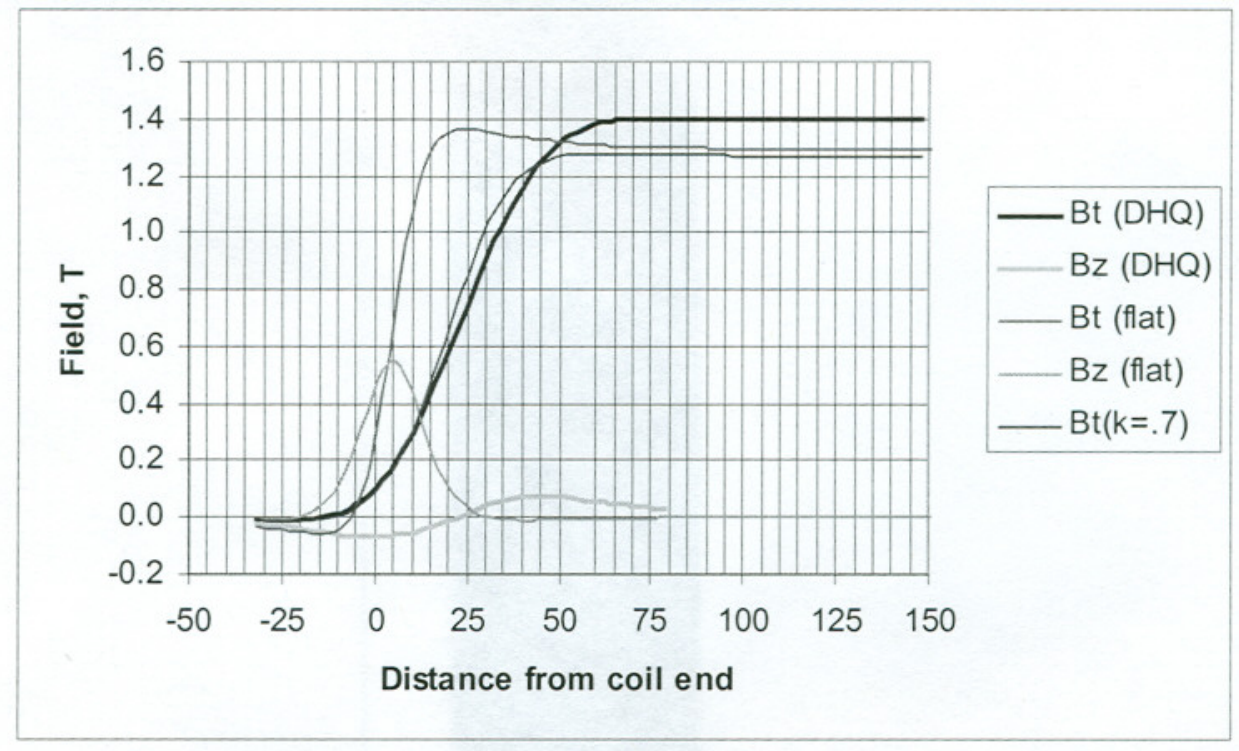

Figure 7. Field plots at $r=16 \mathrm{~mm}$ for a single flat coil quadrupole and a single DHQ showing how the maximum tangential field (Bt) (quadrupole field) and the axial field $(\mathrm{Bz})$ components vary with the distance from the end of the coil.

Calculations were made with CoilCAD ${ }^{\mathrm{TM}}$ [3] using a $3 \times 3$ array of DHQ with a $48 \mathrm{~mm}$ aperture and a length of $300 \mathrm{~mm}$. A complete cross section with all conductor locations and the current directions that were used for such calculations is shown in Figure 8. The relative cell orientation in the array is chosen to yield maximum flux sharing between adjacent cells. 
Figure 8. Cross section of $3 \times 3$ array with DHQ's of $48 \mathrm{~mm}$ aperture and $300 \mathrm{~mm}$ length calculated with CoilCAD $^{\mathrm{TM}}$. The small red and blue circles represent the conductor locations and current directions that are intersected in this plane. The cell size is $73.64 \mathrm{~mm}$. The dashed circles are shown as a scale.

The single cell of the $3 \times 3$ array was modeled with a standard double-helix quadrupole coil. All higher-order multipole fields in a single cell are well below $10^{-5}$ of the main quadrupole field at a reference radius of $75 \%$ of the coil aperture. Using the DHQ cell in a $3 \times 3$ array enhances the field in the central cell due to flux sharing by about $38 \%$. However, the flux sharing in the array assembly has the effect of introducing a dodecapole component into the field of the central cell, which in this case was about $1 \%$ of the quadrupole field at the reference radius.

It should be noted that the flexibility of the DHD design permits the capability of making adjustments to the multipole content of the quadrupole field by adding additional higher harmonic terms to the $\cos 2 \theta$ modulation function of the axial position of the conductor turns. A preliminary calculation using CoilCAD showed that changing this modulation function from $\mathrm{A} \cos 2 \theta$ to $\mathrm{A}\left(\cos 2 \theta+\frac{\cos 6 \theta}{60}\right)$ for all quadrupole cells totally eliminated the dodecapole resulting from flux sharing in the array.

The coil ends of the single DHQ and the array assembly show significant higher-order multipole fields, which cancel to a very high degree when integrated over the full length of the coil. In the integration, the even multipole fields cancel within each end; the skew multipole fields are canceled when the integration is extended over both coil ends.

As for the flat coil design, no attempt was made during Phase 1 to correct the border cells of the calculated $3 \times 3$ array.

\subsection{Edge Termination Scheme}

Several edge termination schemes that had been previously proposed were reviewed and rejected as impractical and / or inadequate. A new edge termination concept proposed by $\mathrm{N}$. 
Martovetsky [4] appears to meet the requirements. In the cross sectional view illustrated in Figure 9, each quadrupole is represented by a square cell, which is framed by blue and red lines of increasing thickness. The color of the lines indicates the current directions; the line thickness is a measure of the local current density. In this 2-dimensional cross section, the assumed current distribution produces quadrupole fields inside of all cells, and the fields in the edge cells and in the interior cells are identical. This ideal current distribution also cancels the external field in the 2-dimensional cross section.

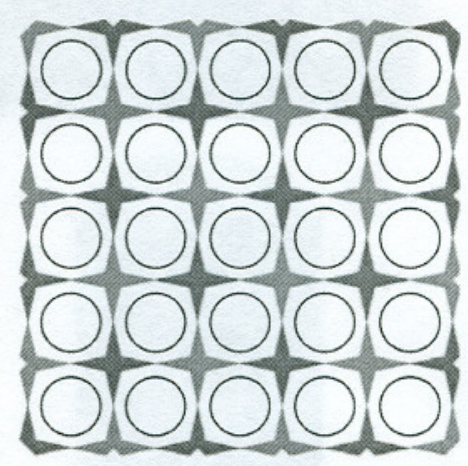

Figure 9.Cross section schematic of current distribution in a 25-cell edge-terminated quad array. (As depicted by N. Martovetsky)

In practice, the peripheral coil windings may be any configuration that approximates the linearly varying current density surrounding each cell. In the array the magnetic flux from a given cell extends into the neighboring cells. This flux sharing reduces the amount of superconductor required for a given field gradient. However, cells at the periphery of the array do not have neighboring cells on all sides and their field strength and uniformity is affected. Thus, a set of coils is needed to adjust the fields in the border cells to the same strength and uniformity as the center cells of the array.

In the case represented in Figure 9, a series of termination coils (with the indicated linearlyvarying current distribution) emulates the presence of neighboring cells. In addition, this solution produces no external field from the array. With such an array termination, all cells have nearly identical boundary conditions electrically and mechanically so that one can test the principal issues associated with arrays by constructing an array with very few elements.

An array design has been worked out that uses the HCX quadrupole design as the basic building block of a $2 \times 2$ quadrupole array. It is based on flat double pancake coils, as shown in Figure 10, to form the four sides of a quadrupole. Each double pancake coil is shared between neighboring cells, as shown in Figure 11. The compact ends of these coils enable the realization of very short quadrupole magnets. 
Advanced Magnet Laboratory, June 2003

DOE SBIR Phase I Final Report: DE-FG02-02ER83359

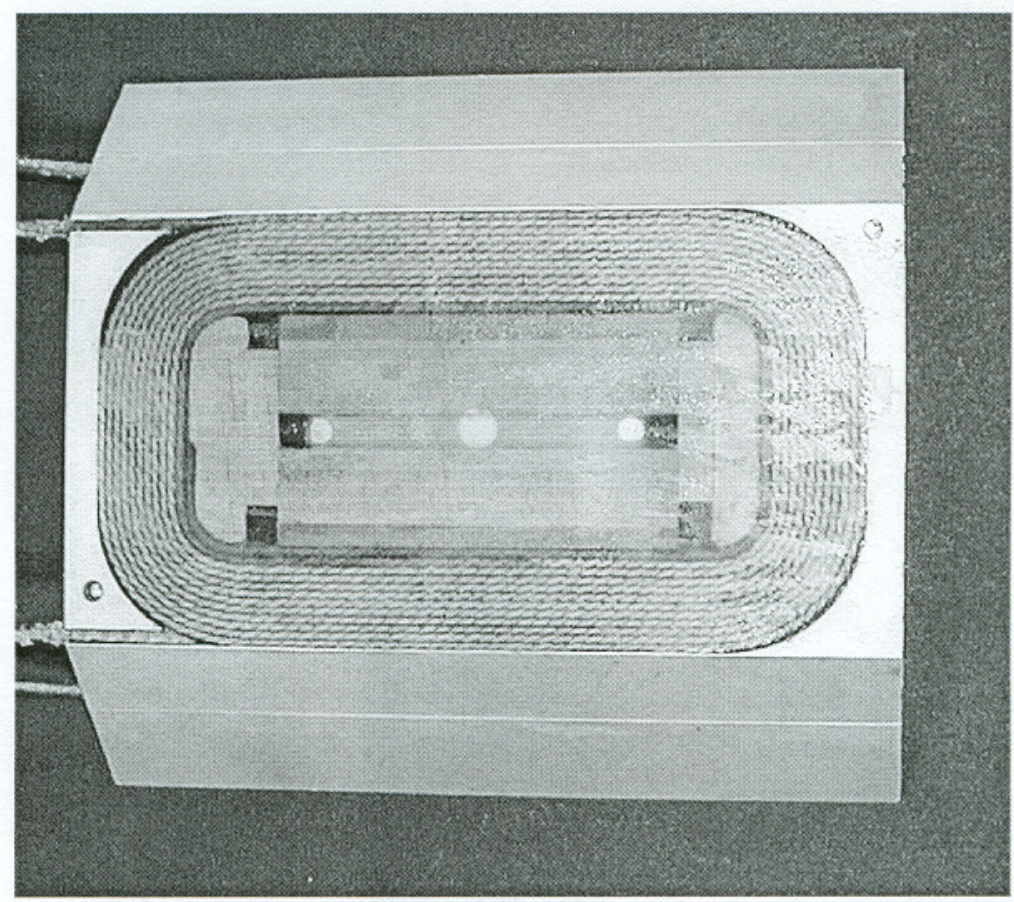

Figure 10. Flat double pancake coil in Al coil holder after vacuum impregnation. (Coil was manufactured at AML for LLNL). Four such coils are arranged in a square box to form an HCX magnet.

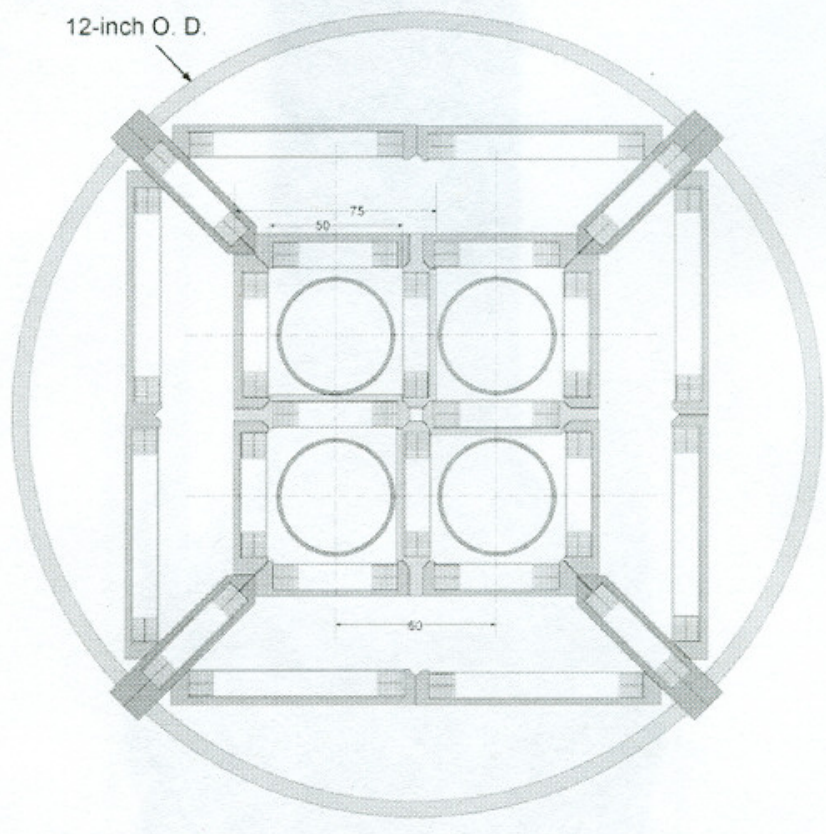

Figure 11. Proposed $2 \times 2$ array with edge termination based on concept by N. Martovetsky. (Auxiliary coil supports are not shown.) 
Target parameters for the central elements of this test array have been developed during discussions with members of the VNL and are listed in Table 3.

Table 3. Some target parameters for edge terminated array test with flat coils.

\begin{tabular}{|l|r|}
\hline \multicolumn{1}{|c|}{ Parameter } & \multicolumn{1}{|c|}{ Value } \\
\hline Aperture between flat coils in array (conductor-to-conductor) & $50 \mathrm{~mm}$ \\
\hline Physical length of array coils & $150 \mathrm{~mm}$ \\
\hline Gradient produced in $2 \times 2$ array coils & $80 \mathrm{~T} / \mathrm{m}$ \\
\hline
\end{tabular}

The conceptual design shown in Figure 11 needs to be fully developed and optimized. In addition to the components shown in the figure, additional coil support structures are necessary to support all flat coils of this assembly in the surrounding shell. All coils of the array cells and the edge termination scheme are based on the flat double-pancake racetrack coils with flat ends housed in a aluminum coil holders, as shown in Figure 10. The 4 coils of the termination scheme, which are in diagonal direction to the array, are fully encapsulated in coil holders to provide symmetrical surfaces for load transfer. A 12 -inch diameter aluminum cylinder with appropriate cutouts for the diagonal coils could be used to house the array and provide external structural support.

The proposed unit has been especially sized to allow testing in one of the vertical magnet test cryostats at LBNL. The main objective of such a test program would be the evaluation of the edge termination scheme.

\subsection{Cryostat Concepts}

The cryostat cost for a quadrupole array is likely to exceed the cost of the superconducting coil assembly. The cryostat design is strongly dependent on the chosen beam tube temperature. A cold beam tube, operated at liquid helium temperature, would yield the lowest rest gas pressure in the beam tube. This would reduce the probability of interactions between beam particles and the rest gas and would reduce energy deposition into the surrounding beam tube. A reduced energy deposition would reduce the cryogenic heat load and therefore the operational cost of the accelerator. Conversely, sufficiently-high energy deposition could increase the temperature of the superconductor and initiate quenches of the quadrupole coils.

Introducing an extra metal shield, a liner, into the beam tube, would reduce energy deposition into the superconducting coils. The liner intercepts radiation originating from the beam and can be operated at an intermediate temperature such as $77 \mathrm{~K}$ to reduce the cryogenic load. Slots in the liner allow rest gas to reach the cold beam tube surface and to get cryo-pumped. The liner, however, would require extra space, and would therefore increase the overall cost of the quadrupole arrays and the whole accelerator. The same cost penalty would result when using a beam tube that operates at room temperature, since extra space between the beam tube and the superconducting coil would be needed for thermal insulation and the helium containment. A room temperature beam tube would also require extra vacuum pumps to maintain the beam tube vacuum, which would lead to a further increase in cost.

Thus, a cold beam tube without liner, if feasible, would lead to the lowest overall cost of the accelerator and at the same time would give the best possible vacuum. 
Each quadrupole array module should have its own cryostat and vacuum system that does not interfere with the adjacent induction modules. Thus it is proposed to cool each array module with forced flow helium in a stainless steel helium containment vessel for which the individual beam tubes form the inner walls of the vessel. The cold beam tube without liner, if feasible, would lead to the lowest overall cost of the accelerator and at the same time would give the best possible vacuum.

\section{TECHNICAL FEASIBILITY/ FUTURE RESEARCH}

Continued R\&D is necessary in several areas associated with the development of suitable quadrupole arrays for multiple beam transport.

a. An edge termination scheme (correction coils) that maintains the same field in the peripheral cells of an array that is produced in the center cells and suppresses the external field of the array.

b. A low-cost solution to the construction of quadrupole array magnets. For instance, the AML double-helix quadrupole (DHQ) design provides relatively simple and easy to manufacture quadrupole magnets with fewer coil interconnections and coil parts.

c. A low-cost cryostat that meets fusion accelerator requirements. This cryostat should include a cold beam tube, which guarantees lowest-possible rest gas pressure in the beam tube without the use of expensive vacuum pumps.

d. Detailed analytical studies would need to be associated as the first stages of these construction projects. Magnet designs would need to be developed, based on type of superconductor cable available. Accelerator and beam studies are needed to optimize parameters of array magnets and cryostats.

\subsection{Edge Termination for Flat Coils}

The quadrupole magnets to be used in a test of an edge-terminated array as shown in Figure 11 could be based on the flat double pancake windings developed by Lawrence Livermore National Laboratory.

All coils in this configuration consist of double pancake windings, i.e., a 2-layer flat coil wound from a single un-spliced length of cable by the method similar to that used to construct the HCX prototype shown in Figure 10. Such HCX prototype quadrupoles have been successfully tested.

By building such an assembly, the key features of an edge termination scheme can be tested in liquid helium in a vertical dewar of a magnet test facility at a National Lab. Properly designed shielding coils, which surround the $2 \times 2$ array, would assure proper quadrupole fields in all four cells and the stray magnetic field of the array would be highly suppressed.

\subsection{Double-Helix Quadrupole Array}

Our analysis indicates that a DHQ design offers the lowest cost solution to the manufacture of quadrupole arrays. To foster the development of low-cost magnet manufacturing technology for fusion accelerators, future $R \& D$ could construct a $3 \times 3$ array of double helix quadrupoles. The field in the central cell of this array is fully surrounded by other quadrupole cells and is therefore representative for the inner cell of a large array. The measurement of the field in this inner cell will verify the field quality of this approach. 
The DHQ is of relatively simple design and easy to manufacture; thus, it is quite feasible to build and test a $3 \times 3$ array of this configuration. This test would investigate the characteristics of such magnets in arrays for more forward-looking applications where longer length elements can be employed. Since the field drop off in the DHQ is more gradual in the ends than in the flat coil design, the magnets in this array will be about $300 \mathrm{~mm}$ long.

The 9 magnets used for this test would be housed in an aluminum box that would fit in a vertical test dewar. A concept of an arrangement of a $3 \times 3$ DHQ array is shown in Figure 12 . This concept shows 4-layer DHQ cells arranged in a $3 \times 3$ array with each coil positioned by composite spacers. The square aluminum alloy box that houses the array also supplies structural reinforcement although the construction of the DHQ is such that they are considered to be selfsupporting.

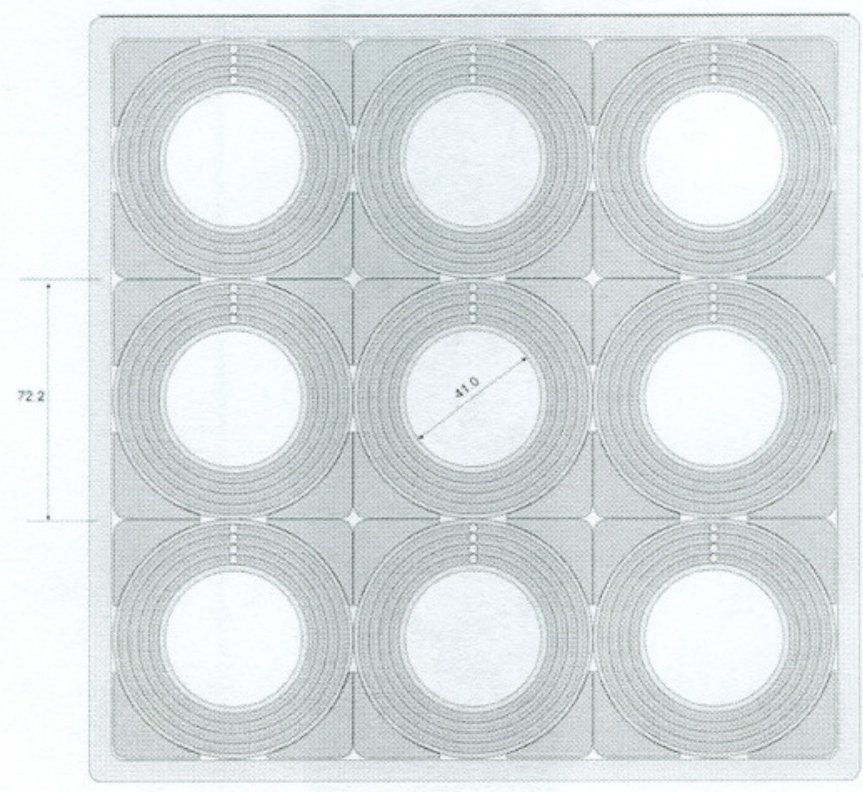

Figure 12. Example of cross-section of a $3 \times 3$ array configuration using double-helix quadrupoles. The aperture of the coils will be selected to match the coil aperture of the flat array quads used in the edge termination array test. Also, the number of coil layers and relative size of conductors and cells may change as a result of the detailed design calculations.

\subsection{Cryostat Design and Construction}

The cryostat cost is a major part of the total magnet cost and in many cases exceeds the manufacturing cost of the superconducting coils. Achieving a sufficiently low beam tube vacuum can be a further cost driver. Using a cold beam tube would guarantee the best possible vacuum and at the same time avoid the cost of additional vacuum pumps. However, the relatively large beam halo expected for a fusion accelerator can lead to high energy deposition into the superconducting coil and compromise magnet performance. In this case a "liner", such as that used in the Large Hadron Collider at CERN, could be required. 


\section{DOE SBIR Phase I Final Report: DE-FG02-02ER83359}

The cold beam tube without liner, if feasible, would lead to the lowest overall cost of the accelerator and at the same time would give the best possible vacuum. In order to test the feasibility of this approach, a special cryostat could be designed for the $3 \times 3$ array of DHQ magnets described in the previous section. The array test measurements discussed in that section would verify operation of all cells before the array is built into its own special cryostat for tests of energy deposition effects.

The individual bores would be kept at the temperature of the surrounding superconducting coil. To test the array performance under these conditions, each quadrupole bore would be capped off and evacuated. A wire would be stretched through the center of each bore, so that applying an electrical current to the wire would simulate energy deposition from beam loss. Since the secondary particle energies from beam - rest gas interactions are rather low, these particles cannot penetrate the beam tube wall and would cause a heating similar to that of the wires.

The cryostat and array with the heater wires would be cryogenically tested, which would yield important data on the allowed energy deposition into the coils. Such data is not currently available, and thus these tests will be critical for determining if a liner is needed for a fusion accelerator.

\subsection{Magnet and Array Design Issues}

There are several distinct areas that require more analytical study.

Since the quadrupole arrays are developed for multiple-beam focusing in induction accelerators, the parameters and design of the magnets need to be optimized for this application. To minimize the cost of these accelerators, it is important to reduce the overall size of the quadrupole arrays, which will be surrounded by accelerating induction cores in the final application. It seems likely that the cell size can be reduced by using elliptical beam tube apertures. Studies should be performed to determine if double-helix quadrupole coils with elliptical aperture can be designed and built.

Progress on edge termination schemes should be reviewed with members of the VNL collaboration. So far no solution has been worked out for the 3-dimensional case of the edge termination scheme and significant end effects are expected for short magnets. Thus, future R\&D should include a complete 3-D analysis of the array edge termination scheme and development of an optimized solution for the construction of a model array to test the scheme. Parameters should be finalized for the aperture, coil length and gradient, and superconducting cable properties of a $2 \times 2$ array of flat coils with the preferred edge termination method. A threedimensional magnetic analysis should provide the basis for selection of the size and geometrical position of the coils in this $2 \times 2$ array. Then, three dimensional calculations for the selected array should be done to predict the multipole field in each of the 4 bores of the array (with and without the edge termination active) and the degree to which the termination coils cancel the external field in three-dimensional space. Tests of an actual $2 \times 2$ array would allow comparing the 3 dimensional field calculations with measurements.

Three-dimensional analysis should also be performed to develop the detailed design for the $3 \times 3$ array composed of double-helix quadrupoles and to characterize the magnetic fields in this array. The distribution of Lorentz forces acting on this configuration should be determined in order to verify that the mechanical design and construction method provides adequate support of coil turns. The three-dimensional fields expected from this type of array can be compared to those expected in the array of flat coils. 


\subsection{Accelerator System Issues}

In order to develop quadrupole arrays for actual accelerators, the beam physics and accelerator interface issues that affect magnet design and performance must be addressed. There are three principal subtasks for such analytical studies.

The first subtask is to calculate where the lost beam particles deposit their energy and the angles at which they strike the various surfaces. The fraction of the lost beam particles that strike the beam tube, compared to the fraction that strike higher temperature surfaces such as the outer surface of the cryostat or the acceleration gap structure, must be determined. The determination of the angles at which the particles strike the surface is important because it determines the number and spectrum of the particles that are created by the collision. This information is needed to determine how much pumping must be provided to prevent the vacuum instability. The calculations needed for this subtask would be done using Monte Carlo techniques that generate and track simulation particles lost from the beam. Tracking the particles requires the magnetic and electric fields in a realistic transport lattice. The capability to design the lattice and calculate the fields was developed as part of the AML Phase I activity.

While the calculations in the first subtask can determine the fraction of lost particles that strike various surfaces, they cannot determine the actual number of particles that are likely to be lost. There are at least three loss mechanisms: collisions between the beam particles and the residual gas in the beam pipe, collisions among the beam particles themselves, and dynamical effects that produce beam halo. In the case of a heavy-ion induction linac, the first mechanism will likely be dominant. Since the magnitude of the effect produced by this mechanism is proportional to the pressure in the beam pipe, detailed calculations of expected pressure are required. The geometry of a multi-beam induction linac is complicated compared to the geometry of most accelerators. Again in this case, Monte Carlo calculations (of molecular transport) are the appropriate approach.

To determine the total refrigeration requirements, the heat load calculated from the results of the first two subtasks must be combined with the heat loads arising from other sources such as radiation and conduction.

Completion of the three subtasks will enable a decision about the necessity of a liner and, if a liner is needed, its temperature and perforation factor (fraction of total area allocated to slots). Completion of the subtasks will also enable one to determine the extent to which the vacuuim instability is a concern.

\section{REFERENCES}

1 N. Martovetsky, B. Manahan, "Main Parameters of the LLNL6r2 design (optimized HCX quad)", January 2002, VNL collaboration online document HCX-MAG-02-0131-01.

2 R.B. Meinke, C.L. Goodzeit, M.J. Ball, "Modulated Double-Helix Quadrupole Magnets", paper 4LC08, ASC 2002, Houston TX, August 2002; IEEE, Transactions on Superconductivity, June 2003. 
3 CoilCAD $^{\mathrm{TM}}$ is a Magnetostatic solver program developed by AML that integrates the Biot Savart Law along space curves. It is particularly useful for solving coils of arbitrary geometry in three-dimensional space.

4 N. Martovetsky, LLNL, March 2003, Edge Termination Scheme diagram, private communication 


\section{Parametric Study of Methods of Beam Optimization in Quadrupole Arrays Roger Bangerter}

\section{FOREWORD}

The first task of the Phase I proposal was to determine the specifications of the array, e.g., the aperture, length, and gradient of the individual quadrupoles in the array and the number of beams in the array. This task proved to be far more difficult than originally anticipated but completing the task led to a major unanticipated benefit, namely, the development of a new computer code MAG3D that integrates beam dynamics, magnetic field calculations with boundary conditions appropriate for quadrupole arrays, and important aspects of mechanical engineering. As the name indicates, the code calculates 3 -dimensional fields. It includes optimizing routines that adjust the important variables such as aperture, length, and field to optimize a selected figure of merit -- subject to essential constraints on ion beam stability and mechanical feasibility. In this sense the code is a major first step toward a detailed accelerator optimization and design code. Section 3 gives a more detailed description of MAG3D and Section 4 gives the important results. The remainder of this section describes the considerations involved in the choice of array specifications and explains why it was difficult to arrive at these specifications.

At the outset it became apparent that the specification of parameters is a controversial subject. Arguments regarding the appropriate number of beams in a fusion accelerator and the aperture of each individual beam go back approximately 20 years. In consulting with various members of the Virtual National Laboratory (VNL) for Heavy Ion Fusion the situation became even more confusing because of conflicting views about the goals of the AML SBIR activity. Some felt that the emphasis should be on parameters that match current designs for the VNL's proposed Integrated Beam Experiment (IBX), a near-term experiment. Others felt that the parameters should be chosen to match future proposed experiments such as the Integrated Research Experiment (IRE). Still others felt that it was appropriate to look at power-plant parameters. Based on DOE guidelines and additional discussions with VNL scientists and management, we concluded that the SBIR effort was not appropriately part of the IBX project. Therefore our research has emphasized parameters for the long-term applications.

Even for long-term applications, the uncertainties in such basic parameters as quadrupole aperture are striking. If we include electrostatic as well as magnetic systems, estimates of optimal aperture size by different researchers have differed by nearly two orders of magnitude (of the order of $1 \mathrm{~mm}$ to $10 \mathrm{~cm}$ ). Because of these uncertainties and the importance of choosing the correct parameters, we now discuss the issues that are involved. We will conclude, not surprisingly, that there are uncertainties in physics and engineering that must be resolved in order to develop firm optimal specifications. Consequently, we have needed to make some judgements regarding the various uncertainties. 
Appendix I

\section{DISCUSSION}

\subsection{Quadrupole Aperture Considerations}

Many of the important issues can be illustrated by considering the familiar envelope equations [1]. These equations give reasonable approximations of the relationships among beam size, focusing strength, unnormalized emittance $(\varepsilon)$, and perveance $(Q)$ :

$$
\begin{aligned}
& a^{\prime \prime}=k^{2}(z) a+\varepsilon^{2} / a^{3}+2 Q /(a+b) \\
& b^{\prime \prime}=-k^{2}(z) b+\varepsilon^{2} / b^{3}+2 Q /(a+b)
\end{aligned}
$$

where $a$ and $b$ are the transverse semi-axes of the beam (assumed to be elliptical in a quadrupole transport system) and the primes indicate differentiation with respect to the longitudinal coordinate $z$. The quadrupole strength is given by $k^{2}=G /(B \rho)$ where $G$ is the magnetic field gradient and $B \rho=p / q e$ is the magnetic rigidity of the beam where $p, q$, and $e$ are respectively beam momentum, beam charge state $($ often +1$)$ and the electronic charge. The sign of $G$ alternates from quadrupole to quadrupole but for purposes of illustration, we can ignore the alternation and replace $k$ with an average wave number $\sigma_{0} /(2 L)$ where $\sigma_{0}$ is the undepressed phase advance (undepressed tune) in a lattice period of length $2 L$ where $L$ is the length between the centers of adjacent quadrupoles. By undepressed we mean the phase advance of a single particle in the absence of space charge. The replacement of $k$ by an average wave number is referred to as the smooth approximation. In the smooth approximation, we also replace $a$ and $b$ with an average beam radius $r$ obtaining the single envelope equation

$$
r^{\prime \prime}=-\sigma_{0}{ }^{2} /\left(4 L^{2}\right) r+\varepsilon^{2} / r^{3}+Q / r .
$$

For a matched beam with constant radius both $r^{\prime \prime}$ and $r^{\prime}$ are equal to zero. Moreover, throughout most of an accelerator for heavy ion fusion, the second term on the right of (2) is significantly smaller than the third term. In other words, the beams are spacecharge dominated rather than emmitance dominated. In high energy accelerators the beams are usually emittance dominated. Dropping the second term in (2), gives $Q=\left[\left(\sigma_{0} r /(2 L)\right]^{2}\right.$ The perveance is proportional to the beam current $I$. Nonrelativistically it is given by $Q=30 I q^{2} /(T \beta)$ where $I$ is in amperes, $T$ is ion kinetic energy in $\mathrm{eV}$, and $\beta$, as usual, is the speed of the ions divided by the speed of light. These equations for $Q$ show that the transportable beam current increases with increasing $\sigma_{0}$. It is often desirable to transport as much current as possible but there is an important limit on $\sigma_{0}$. Specifically, if $\sigma_{0}$ is larger than 80 to 90 degrees, space-charge dominated beams develop serious instabilities. The quantity $\sigma_{0}$ is approximately given by $\sigma_{0} \approx G L^{2}$ $\eta /(B \rho)$ where $\eta$ is the occupancy factor, the effective magnetic length of a quadrupole divided by $L$. The equality becomes exact in the limit of small $\sigma_{0}$ and small $\eta$. Readers who are interested in more precise analytic expressions may refer to [2]. Setting $\sigma_{0}$ to its maximally allowed value and combining the equations just described, we obtain an important result: 


$$
I_{\max } \propto G r^{2}=B r^{2} / X
$$

where $I_{\max }$ is the maximal current that can be transported and $B$ is the quadrupole field measured at radius $X$. This result is hardly remarkable from a physics standpoint. The radial electric field at the edge of a cylindrical beam is proportional to $I_{\max } / r$ (Gauss's Law). This field must be balanced by the magnetic field which is proportional to $G r$. While result (3) is not remarkable from a physics standpoint, it is remarkable for the effect that it has had on the heavy ion fusion program. Considerations relating to (3) are critical considerations in arriving at optimal specifications for a quadrupole array. They are also at the heart of the controversial aspects of this problem. Many of the important aspects of the problem and the controversy surrounding the problem first arose in the context of electrostatic quadrupoles. For electrostatic quadrupoles, one replaces $B$ with $E / \beta$ where $E$ is the electric field.

All researchers agree that the average beam current density $J_{\text {ave }}$ defined as the total beam current in the array divided by the total transverse area of the array is an important figure of merit. Consider induction linacs, the case of current interest in the U.S. heavy ion fusion program. If the area of the arrays is large for a given beam current, the induction cores that surround the arrays must also be large, leading to high cost for both the cores and their associated pulsers, and to low efficiency. Low accelerator efficiency has a negative effect on the recirculating power fraction in a fusion power plant and leads to increased capital cost for the entire plant. For now we adopt the point of view that maximizing $J_{\text {ave }}$ leads to optimal array specifications.

If one scales all transverse dimensions of a system, for example $r$ and $X$, by a factor $s$ while holding the field fixed, equation (3) shows that the current scales linearly with $s$, but the quadrupole area scales as $s 2$. Taken at face value, this implies that one can obtain arbitrarily large $J_{\text {ave }}$ by scaling to arbitrarily small size - at fixed field. For electrostatic systems, the attainable electric field is approximately independent of $s$ for scale sizes less than about $10 \mathrm{~mm}$ and decreases with increasing size for sizes greater than about $10 \mathrm{~mm}$. Thus, for electrostatic systems, equation (3) implies that the optimal quadrupole aperture is very small indeed. Unfortunately, there are important constraints. It is well known that quadrupole-to-quadrupole transverse alignment errors lead to inevitable beam misalignment and that this problem is worse for small apertures. The aperture must clearly be larger that the misalignment.

Andris Faltens and his coworkers at Berkeley adopted a simple linear approximation to account for misalignment. Specifically, they assumed that the beam would be misaligned by an amount $\delta$. In addition, numerical simulations showed degradation of beam quality (emmitance growth) unless the aperture radius $R$ exceeded a certain factor $\alpha$ times the major radius of the beam $a$, usually about $125 \%$. Combining the misalignment assumption with the results from the numerical simulations leads to

$$
R=\alpha a+\delta
$$

We can combine (3), (4), and the definition of $J_{\text {ave }}$ to obtain 
Appendix I

$$
J_{\text {ave }} \propto(R-\delta)^{2} / R^{3}
$$

where we have assumed that $R \propto X$ and $a \propto r$. We have also assumed that the transverse area occupied by a quadrupole is proportional to $R^{2}$ and that the field is constant. The first three of these assumptions are excellent approximations for electrostatic quadrupoles and the constant field assumption can easily be removed by writing a slightly more complex expression where the field varies as some power of $R$. Our goal is to obtain the simplest expression that illustrates the basic issues. Note that (5) is independent of $\alpha$.

We maximize $J_{\text {ave }}$ by differentiating (5) with respect to $R$ and setting the derivative to zero. This procedure gives

$$
R=3 \delta
$$

In summary, the optimal aperture is three times the fixed spacing that is allowed between the beam edge and the aperture.

So far, in the spirit of a simple model that illustrates the issues, none of this is particularly controversial. The problem we encountered is the fact that different researchers have vastly different views about the proper value of $\delta$. These arguments have persisted for nearly 20 years. Some argue that it is impossible to build and align the quadrupoles accurately and believe that it is prudent to set $\delta$ to approximately $10 \mathrm{~mm}$. Others argue that one can either build and align accurately or sense and correct the beam position so that the appropriate value of $\delta$ is very small compared to $10 \mathrm{~mm}$. The advocates of $\delta \approx 10 \mathrm{~mm}$, argue that accurate beam sensing and correction are impossible or expensive in the presence of the noise caused by the induction cores and pulsers. In response, those who believe in small $\delta$ point out that the Livermore researchers were able to measure the beam in their Small Recirculator, an induction accelerator, to a precision of the order of $100 \mu \mathrm{m}$ in the presence of noise [3]. In the Small Recirculator both the beam current and the noise were smaller than they would be in a power plant driver $\underline{\text { so }}$ the issue has never been fully resolved in the proper environment and large differences in opinion remain.

It is noteworthy that one of the original principal goals of the HCX (High Current Experiment) at Berkeley was the resolution of this important and longstanding issue[4].

Because of funding limitations and other issues, it is not clear that the HCX will be able to achieve this goal. We recommend that the DOE solicit new SBIR proposals to develop beam diagnostics, correction elements, and signal processing algorithms to resolve the issue. In this regard, Section 4 presents a detailed discussion of the validity of optimization procedures typified by equations (4) through (6) and suggests needed research.

\subsection{Array Design Considerations}

We now turn to specifications for magnetic quadrupoles. Here the some of the assumptions leading to (6) break down. In particular, Ampere's Law $\int B \cdot d l=\mu_{0} I_{C}$ where $I_{C}$ is the current in the conductors, limits the scaling to small size that appeared attractive from equation (3). Here we have used $\mu_{0}$ because magnetic materials such as 
iron are of limited use in an array. The individual quadrupoles are surrounded by other quadrupoles rather than by an iron yoke that could serve as an effective flux return region. For a given current density in the conductors, $I_{C}$ scales as the square of the quadrupole scale factor $s$ because it is proportional to the conductor area. On the other hand, $\int B \cdot d l$ scales only linearly with $s$ so that the $B$ that can be attained, increases with increasing s. $B$ scales as $s$. If the thickness of the conductor shell is small compared to the quadrupole aperture, a constant thickness is required to maintain constant $B$. The interested reader can easily include this additional detail in (5). The net result is that the optimal aperture increases by an amount comparable to the thickness of the conductor shell. Since we do the optimization more accurately using numerical methods, we will not derive this result here.

Some early researchers assumed that superconducting quadrupole systems would require warm-bore construction with approximately $10 \mathrm{~mm}$ of multi-layer insulation. This assumption was originally reasonable. Beam loss owing to beam-gas and beam-beam collisions will heat the beam tube leading to a large refrigeration load if the superconductor is not insulated from the beam tube. If one adds $10 \mathrm{~mm}$ of insulation and structure to $\delta=10 \mathrm{~mm}$ from misalignment, multiplies by the factor of three from (6) and adds the contribution from the superconductor and its structure, one obtains optimal aperture diameters $>120 \mathrm{~mm}$. Although such aperture diameters are large compared to those of modern high energy accelerators, they were commonly assumed to be optimal for heavy ion fusion. For example, several years ago these large diameters were given to $\mathrm{AML}$ as specifications for another study.

By using vacuum-tube liners operating at a temperature between ambient temperature and superconducting temperature, such as in the Large Hadron Collider, we now believe that no multi-layer insulation is needed. Section 4 explains the considerations leading to this conclusion. (Detailed calculations of heat transfer and cryostat design will be part of the Phase II work.)

Elimination of the warm-bore in favor of a liner at intermediate temperature substantially reduces the optimal aperture. It also simplifies the construction of the array. More importantly, it provides more space for beam so that for a given conductor geometry it greatly increases the average beam current density. Unfortunately, there are additional new considerations that tend to reduce the average beam current density. These new considerations may also affect the optimal aperture size.

Beam halo and its effect on the production of unwanted electrons is one relatively recent consideration. Beam halo effects may place an additional limit on the ratio of beam radius to aperture - the so-called fill factor or dynamic-aperture factor. For example, if these effects were to limit the fill factor to $50 \%$ rather than the $80 \%$ corresponding to $\alpha=1.25$, it would have a negative effect on beam current density. It would probably also reduce the optimal aperture. The reason that optimization of (5) leads to $R=3 \delta$ is because (4) severely limits the fill factor at small apertures but leads to large fill factors a large apertures. For example, if $R$ were $11 \mathrm{~mm}$ and $\delta$ were $10 \mathrm{~mm}$, the fill factor would be less than $10 \%$. In contrast, for large apertures, the fill factor approaches $1 / \alpha$. Thus equations (4) through (6) lead to a situation in which large apertures are more conservative than small apertures in terms of beam alignment, but are less conservative in terms of fill factor. In any case, halo physics and its effect remains 
an important uncertainty in determining optimal specifications. In this study, we have simply limited the fill factor to $60 \%$ for our base-case specifications. We have also chosen $\delta=5 \mathrm{~mm}$ as our base case. This value is approximately the midpoint of the extreme views and should allow us to scale the technology that we develop either way.

We now turn to a detailed description of the process used to set the array specifications. The ultimate figure of merit for a fusion power plant is cost of electricity (COE). Quadrupole apertures, fields, and number of beams should be chosen to optimize (minimize) COE. Performing a believable systems study to determine the proper specifications for this contract lies far outside the scope of the contract and is probably not possible at the present time because of important uncertainties in physics and engineering.

Consequently, we adopted maximization of $J_{\text {ave }}$ as our primary criterion. This criterion was adopted in consultation with the VNL. Most beam physics considerations will likely favor smaller apertures that those given by maximization of $J_{\text {ave }}$ but, for a given total beam current, it may be less expensive to fabricate fewer larger quadrupoles. Nevertheless, maximization of $J_{\text {ave }}$ is certainly a reasonable criterion at this time. This criterion was augmented by an additional criterion suggested by the VNL, namely that the higher order coefficients in a multipole expansion of the magnetic field, integrated over the length of the quadrupole, be less than than $1 \%$ of the quadrupole $(n=2)$ coefficient. In practice, we are able to satisfy this criterion by choosing coil configurations that zero the dodecapole $(n=6)$ coefficient. This coefficient has the lowest value of $n$ (other than $n=2$ for conductor configurations with quadrupole-like symmetry.

\section{OPtimization METHOD WITH MAG3D}

In our simple example leading to equation (6), we varied only one quantity, namely the quadrupole aperture. In general a beam and its associated quadrupole must be described by a relatively large number of variables and parameters. Figure 1 shows the transverse $(x-y)$ cross section of one quadrant of an idealized beam and quadrupole. If we temporarily ignore the beam and assume that the curved surfaces are elliptical, one can draw Figure 1 by specifying the following:

1. The major and minor radii of the liner (the aperture)

2. The thickness of the liner in two directions (the directions of the major and minor radii)

3. The gap between the liner and the vacuum tube in the two directions

4. The thickness of the vacuum tube in the two directions

5. The gap between the vacuum tube and the inboard structure

6. The thickness of the inboard structure

7. The thickness of the insulation between the conductor block and the structures

8. The thickness of the conductor block

9. The $y$-coordinate of the bottom of the conductor block

10. The $y$-coordinate of the top of the conductor block

11. The thickness of the outboard structure 


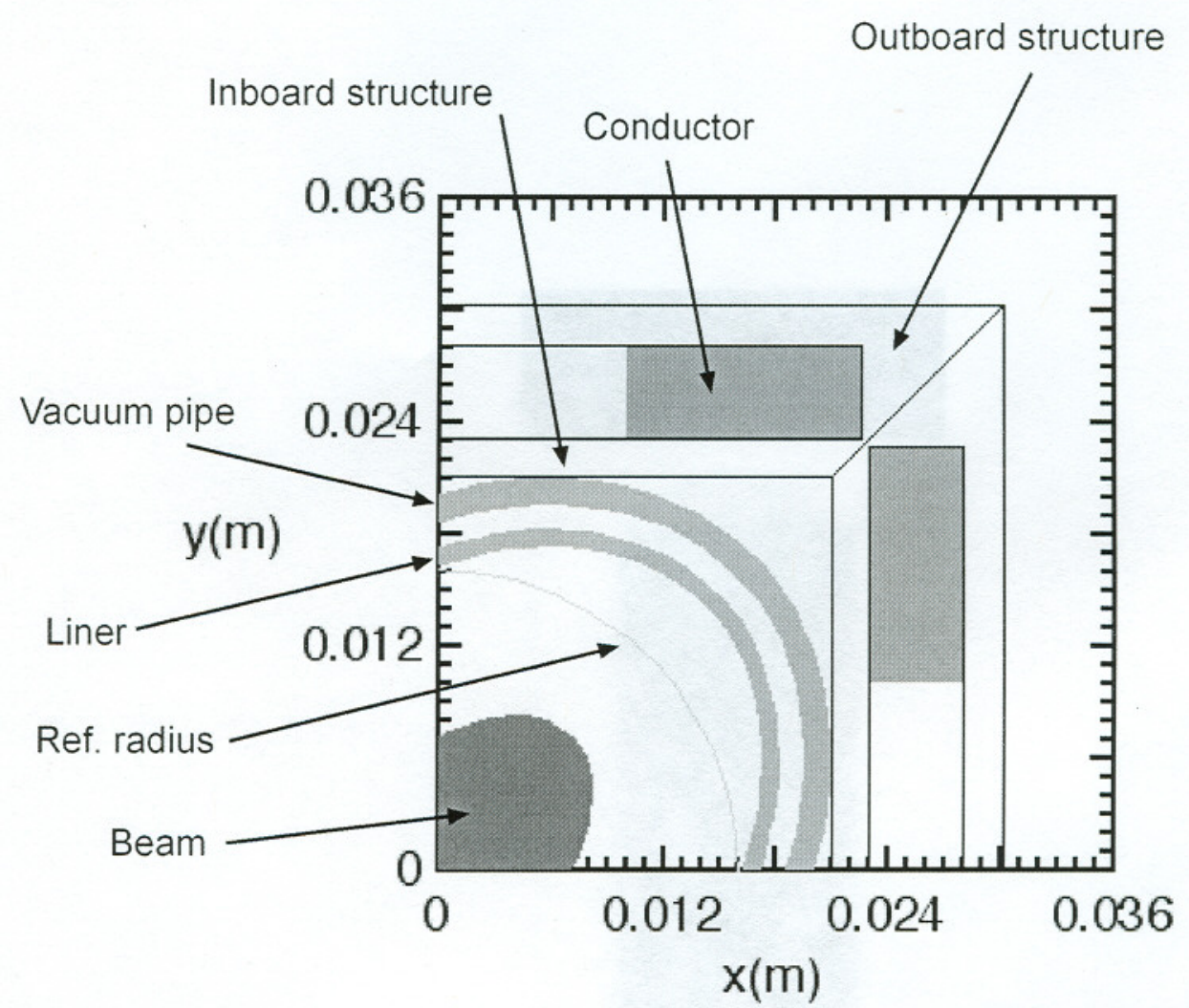

Figure 1. Transverse (x-y) cross section of one quadrant of a quadrupole. The circle marked "reference radius" is the radius at which the multipole expansion of the field is calculated. In principle one could have a gap between the vacuum pipe and the inboard structure but none is shown. Also, for simplicity, the electrical insulation surrounding the conductor is not shown.

The third dimension adds additional variables. Figure 2 shows the $y-z$ cross section. Here one must additionally specify the following:

12. The length of the straight section of the conductors

13. The radius of curvature of the innermost conductor surface

14. The thickness of the end structure

15. The gap between the end structure and the end of the cryostat

16. One half the acceleration gap 


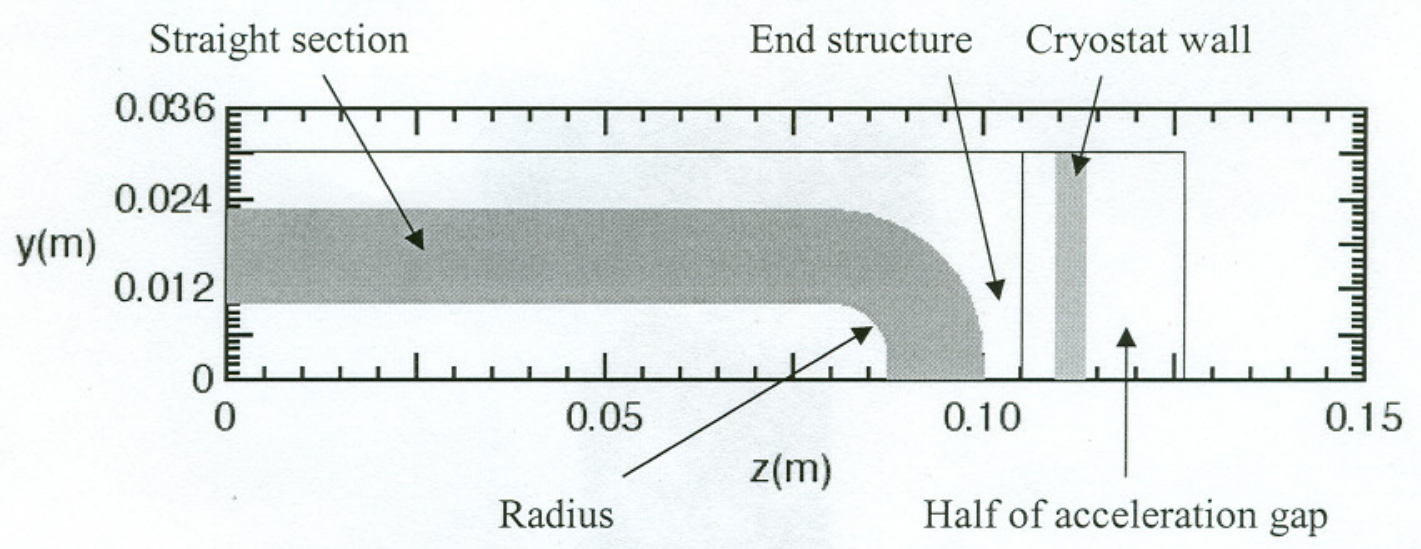

Figure 2. Longitudinal (z-y) cross section of a quadrupole.

The conductor block consists of a number of cables separated by insulation. It can be specified by:

17. The number of cables

18. The current in each cable

So far we have specified 22 numbers. These variables, augmented with appropriate boundary conditions, are sufficient to calculate the 3-dimensional magnetic field of the quadrupole. Not all the variables are independent. In the most general case, the structures must be thick enough to support magnetic forces, helium pressure in even of a quench, pre-stress forces, etc. The thickness of the structures also depends on variables such as their radii or length. Additionally, the magnetic field depends on the current, but the current that can be carried by a given conductor also depends on the magnetic field. Moreover, as discussed in Section 2.1, beam stability imposes a constraint on the phase advance. Finally, the variables must be chosen to give adequate field quality. As will be discussed in more detail below, we solve the problem of interdependence by varying all important variables subject to a number of constraint equations. The constraint equations are, in general, nonlinear.

Now consider the beam. Here we set the mass, charge, and kinetic energy. We must also specify the beam emittance. This can be done directly or by specifying some equation or model that relates beam current and emittance, e.g., a specification of the beam brightness. Finally we require some equation, an analog of equation (4), that relates the major radius of the beam to the aperture that has been specified above. At this point, we can use the coupled differential equations (1) to solve for the minor radius of the beam and its perveance (or equivalently, its current). This procedure requires an additional condition, namely that the solution of (1) be a matched solution, a solution that repeats every lattice period of length $2 L$. Given the beam current and the size of the 
quadrupole as specified above, we can compute our figure of merit $J_{\text {ave }}$. There are more variables than constraints, so the variables can be adjusted to maximize the figure of merit.

The MAG3D code used to perform the optimization has the following features:

1. Field calculations. Currently MAG3D uses Fast Fourier Transforms (FFTs) to solve $\nabla^{2} A=-\mu_{0} j$ where $A$ is the vector potential and $j$ is the conductor current density on a 3-dimensional mesh. A numerical evaluation of $B=\nabla \times A$ using centered derivatives gives the magnetic field. The conductors are approximated by a large number of current elements of small but finite length. The current density corresponding to each element is assigned to the eight closest grid points (the corners of the parallelepiped containing the center of the current element) using a linear weighting procedure. FFTs were chosen partly to facilitate the application of boundary conditions appropriate to a quadrupole in an array. The arrays are terminated transversely in such a way that all quadrupoles have the same boundary conditions. Each quadrupole is contained in a unit cell consisting of a rectangular parallelepiped of length $L$ whose ends are squares of width $2 H$. The boundary conditions are chosen to enforce quadrupole-like symmetry, the magnetic field lines are perpendicular to the surfaces of the parallelepiped, and the boundary conditions are periodic - a condition that is natural to implement using FFTs. We define quadrupole-like to mean that all octants are replicas or a mirror images of each other. As the individual quadrupoles fill more and more of the cell, the flux sharing among neighboring cells becomes stronger. In the limit, this flux sharing leads to a factor of two enhancement in magnetic field, for a given conductor current, relative to a quadrupole in free space. This is the same factor that would be obtained by surrounding the quadrupole with a "perfect" iron (very high permeability) flux return yoke and explains why iron is of limited utility in an array. It is also interesting that, unlike a single quadrupole, a $\cos (2 \theta)$ current distribution does not give perfect quadrupole fields.

2. Ion beam calculations. The magnetic fields, computed as described above, are used with leapfrog integration to solve the equations of motion for two independent ion trajectories to obtain the undepressed phase advance $\sigma_{0}$. The computed magnetic fields and leapfrog integration are also used to solve equations (1). Newton's method with numerical derivatives is used to ensure that the solution of (1) is a matched solution.

3. Constraints. As previously explained, there are several important constraints. The constraints relating magnetic field and conductor current are particularly simple. We currently use $i=A_{S C} F_{S} j_{S C}\left(B_{\max }\right), i=A_{C u} j_{C u}$, and $A_{S C}+A_{C u}=A_{T o t}$ where $i$ is the current in a conductor and $A_{T o t}, A_{S C}$, and $A_{C u}$ are respectively the total area of the conductor, the area of the superconductor, and the area of the copper. The total area is really an effective area that takes into account such things as the packing fraction of the cable and other geometric factors in the cable. A safety factor $F_{S}$ is applied to the superconductor current density $j_{S C}\left(B_{\max }\right)$, which is an analytic or numerical function 
of the maximal magnetic field $B_{\max }$ seen by the superconductor. These equations are augmented with one additional condition obtained by solving the field equations. The field equations are initially solved assuming some specified current, say $1 \mathrm{~A}$. The maximal field at any point on the mesh is located and used to find the coefficient relating $B_{\max }$ and $i$. The equations are algebraically combined into a single nonlinear equation that is solved numerically. Solution of these equations gives the magnitude of the field at the highest possible operating current, the copper-to-superconductor ratio, etc. The calculation of $\sigma_{0}$ is complicated since it depends on nearly all the other variables. The constraint is, however, very simple: $\cos \left(\sigma_{0}\right)=$ a specified constant. We use $\cos \left(\sigma_{0}\right)$ rather than $\sigma_{0}$ because the absolute value of the calculated value of $\cos \left(\sigma_{0}\right)$ can exceed 1 . This simply means that the beam is unstable, a situation that can occur before the constraint procedure has converged. The constraint on field quality is applied by nulling or minimizing some function of the coefficients of a multipole expansion of the magnetic field. The other main constraints involve geometry and mechanical engineering. They fall into two categories: those that involve the magnetic field and/or the solutions of equations (1) and those that do not. The equations that do not are used directly to reduce the number of variables. For example, the thickness of the liner and the gap between the liner and the vacuum pipe appear to be governed more by thermal considerations than by the magnetic field. (The liner must, of course, be robust enough to withstand magnetic forces arising from a quench.) We directly calculate the thickness of the liner and the gap from the major and minor radii of the liner. The relationship between the liner dimensions and the major radius of the beam is another example. We specify three parameters, $\alpha$ and $\delta$ as defined in relation to equation (4) and a third parameter that specifies the maximal fill factor. The clearance between the beam and the liner is determined by an analytic expression that approaches (4) in the limit of small apertures and asymptotically forces the maximal fill factor at large apertures. In some optional models that may be used by MAG3D, the thickness of the inboard structure, the thickness of the outboard structure, and the thickness of the end structure depend on the magnetic field. We leave these three quantities as variables but iteratively adjust them during the optimization process to be equal to the required values calculated using the magnetic fields. We currently use formulas similar to those found in engineering handbooks to calculate the required thickness. This procedure is adequate for our purposes since we check our results with detailed mechanical engineering codes. However MAG3D has been designed to allow the inclusion of multi-dimensional, finite-element engineering algorithms in a straightforward way.

4. Typical optimization procedure. We usually choose the major radius of the quadrupole aperture (major radius of the liner) as one independent variable and plot average beam current density as a function of this variable as shown in Figure 3. We calculate as many variables as possible from constraints that do not involve calculation of magnetic field or beam dynamics. Some variables have a negligible effect on average beam current density and/or can simply be set to reasonable or known values. This procedure normally leaves less than ten variables that must be 
varied to achieve an optimum. For the racetrack example shown in Figure 1, the remaining variables are the thickness of the three structures described in the previous paragraph, the thickness of the conductor block, the $y$-coordinate of the bottom of the conductor block, and the length of the straight section. In this case, the number of constraints is only two less than the number of variables.

Because of the fact that we use discrete Fourier transforms and finite-precision arithmetic, the calculation of the objective function $J_{\text {ave }}$ is noisy at a level of a few tenths of $1 \%$. This is of negligible consequence in terms of the answer but it sometimes makes it difficult to use optimization methods that require numerical derivatives. In these cases, we simply make repeated sweeps over the variables using Brent's Method [4] to hunt down the optimum. Also, for the double-helix, the number of turns is an important optimization variable. In this case also, we do repeated sweeps over the integer variable until we achieve convergence.

A typical optimization requires the calculation of the order of ten-thousand different quadrupole and beam configurations and takes less than an hour on a $450 \mathrm{MHz}$ G4 Apple computer with Altivec vector processing. MAG3D is written in modern Fortran and is compiled using an Absoft Pro Fortran 90/95 compiler.

\section{Parametric Study of Quadrupole Configurations}

In this section we present the results of parameter studies that we used to determine the specifications for our study. We note at the outset that such important parameters as bore size, field strength, and quadrupole length depend, to some degree, on the mechanical design and conductor configuration of the of the quadrupoles in the array. We primarily considered two different conductor configurations, a racetrack configuration using "pancake" coils as illustrated in Figures 1 and 2 and the double-helix configuration shown if Figure 4. Near the end of our study we began the study of one additional conductor configuration that will be described below. We did not have time to optimize this configuration nor to study the details of its fabrication.

We began our study of the racetrack configuration with a robust mechanical design that scaled the structure thickness with both the overall dimensions of the quadrupole as well as with the magnetic field. Figure 3 shows the average beam current density as a function of aperture for this design. As explained in Section 3, each point on this curve represents a design that was optimized by varying the remaining important variables. For this particular case, we assumed that the beam was strongly space charge dominated. This was achieved by setting the normalized emittance to a small value $(0.1 \mathrm{~mm} \bullet \mathrm{mr})$. We will discuss the effects of larger emittance values later in this section. Note that the optimal aperture radius is slightly less than $20 \mathrm{~mm}$. On the basis of this result, we adopted an aperture diameter of $40 \mathrm{~mm}$ as our base case. As explained in Section 2.2, this result was obtained using a compromise value of $\delta$.

The corresponding calculation for the double helix (Figure 4) is shown in Figure 5 
Advanced Magnet Laboratory, July 2003

DOE SBIR Phase I Final Report: DE-FG02-02ER83359

Appendix I

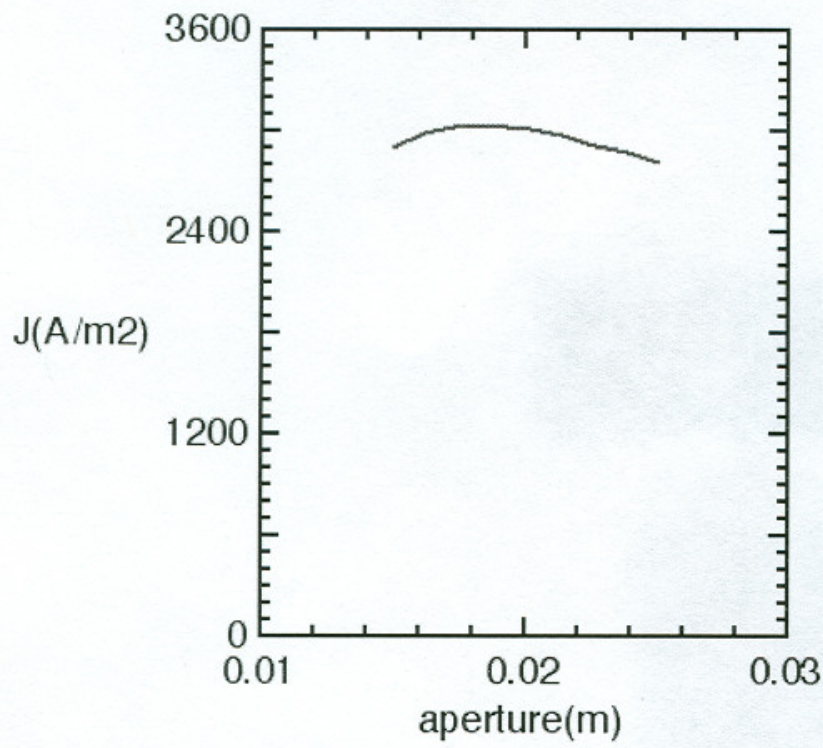

Figure 3. Average beam current density as a function of aperture for a racetrack quadrupole.

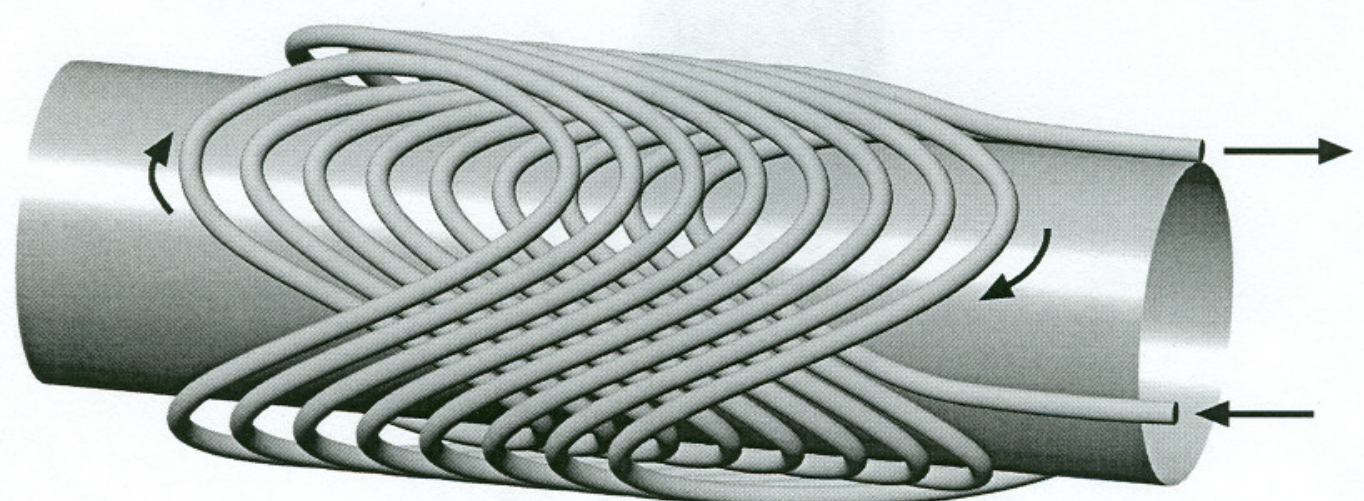

Figure 4. Example of the coil geometry used in the double-helix quadrupole for a 2-layer coil. The current directions shown cancel the solenoid field and add the quadrupole field from each layer. 


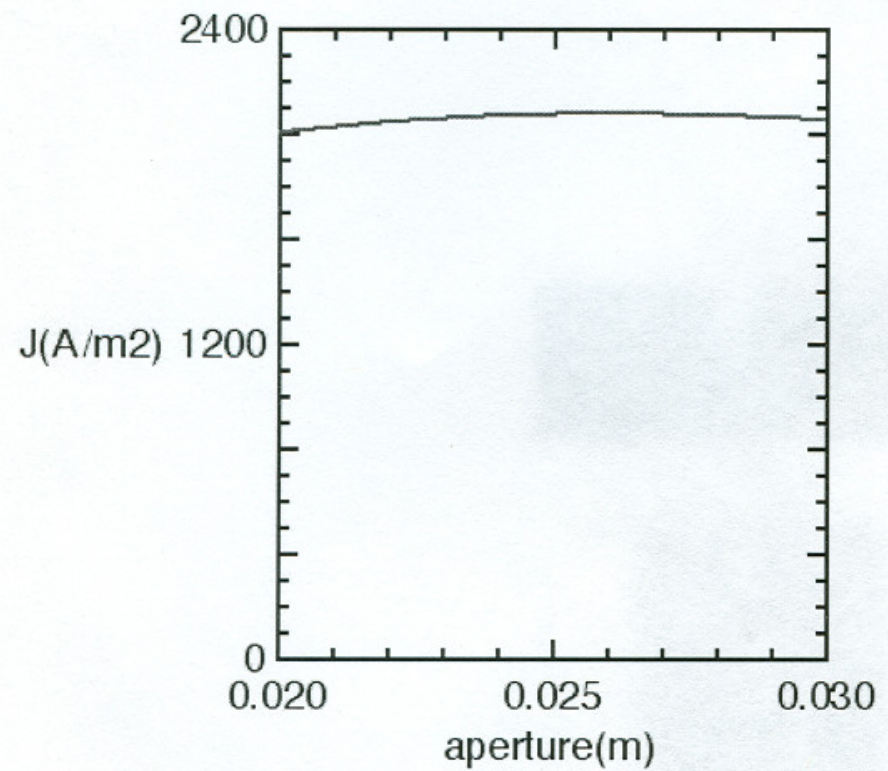

Figure 5. Average beam current density as a function of aperture for a double-helix quadrupole.

This double-helix design has a larger optimal aperture and a lower beam current density than the racetrack. In neither case is there a very strong dependence on aperture so that other considerations may ultimately play important roles in determining the true optimal aperture, for example, the number of beams and fabrication costs. Consider first the number of beams. Figure 6 shows a schematic diagram of an array containing 32 beams.

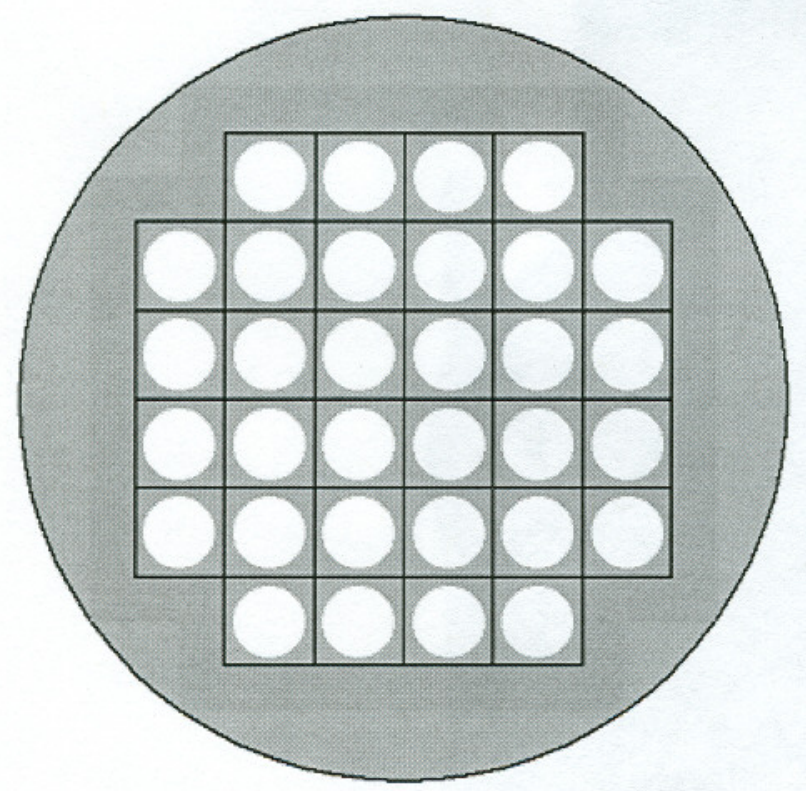

Figure 6. A schematic diagram of an array with 32 beams. 
In general, larger numbers of beams allow one to more nearly approximate the circular aperture of an induction core. Moreover, in an array it is necessary to terminate the transverse magnetic fields with additional conductors. The space required the additional conductors is proportional to the transverse size of the individual quadrupole cells and is typically about half a cell size surrounding the entire array as indicated by the gray region in Figure 6. Thus larger numbers of beams lead to smaller fractional termination space. Figure 7 shows two different figures of merit for arrays containing from 1 to 256 beams.

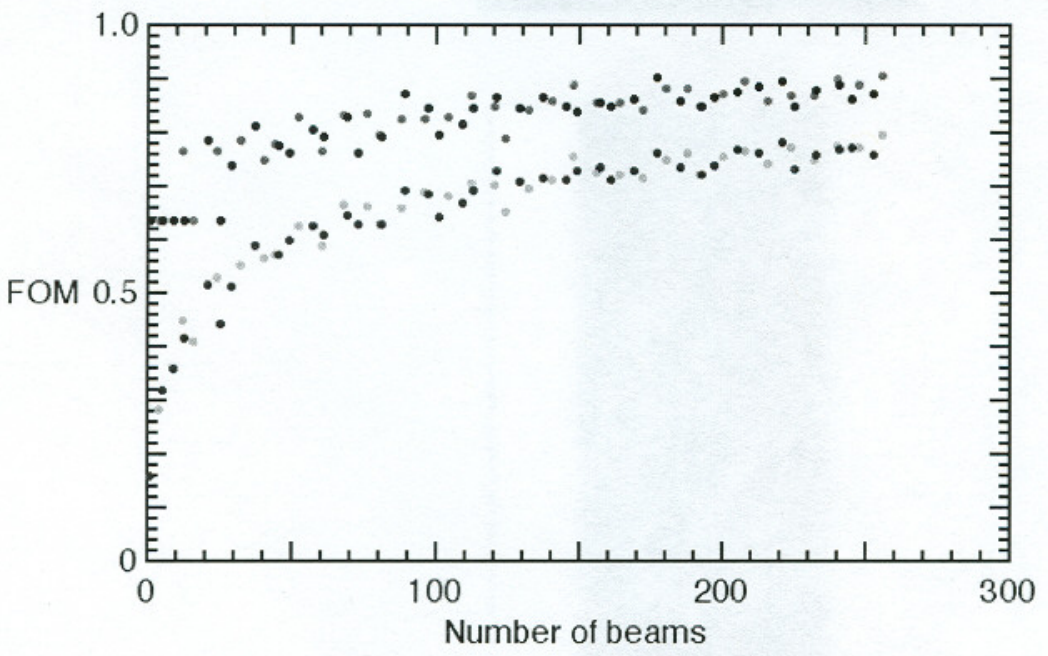

Figure 7. Packing figures of merit for different numbers of beams. Odd numbers of beams are denoted by blue and black dots. Even numbers of beams are denoted by green and red dots. The green and blue dots include the effect of field termination.

There are two sequences, a sequence containing odd numbers of beams and a sequence containing even numbers of beams. The odd sequence is formed by starting with one beam and adding 4 or 8 beams at a time in a way that minimizes the radius of a circle surrounding the beams. The even sequence starts with 4 beams. The top two sets of points give a figure of merit that is the fraction of the area of a circle that is occupied by the beam cells. The lower sets of points include the effect of the field termination. Note that there are "magic numbers" of beams that have good packing properties. For a given total beam current, the number of beams increases with decreasing aperture size. If this effect were included in our calculations, it would push the optimal apertures to smaller size. For relatively small numbers of beams, say less than 32 , this effect is a large effect. For large numbers of beams it is less important. On the other hand, fabrication costs will likely increase with increasing beam number so inclusion of this effect would argue for larger apertures. As we had agreed at the beginning of our study, we ignored all effects except optimal beam current density.

There are other, better mechanical designs than the design used for Figure 3. The racetrack quadrupole currently under construction at AML does not require so much inboard structure. Inside the conductors it has only a circular vacuum pipe that is thick enough to hold the helium pressure in the event of a quench. An elliptical beam pipe can be used instead of a circular beam pipe since the beam itself is elliptical. Since the 
direction of the major axis of the beam ellipse alternates direction from quadrupole to quadrupole, the major axis of the beam pipe must also alternate. This does not introduce much increased complexity because there are acceleration gaps between the quadrupoles so that the beam pipe cannot be continuous in any case. The use of an elliptical pipe allows one to reduce the cell size for a given aperture and leads to a higher average beam current density. Figure 8 illustrates a design with an elliptical beam pipe.

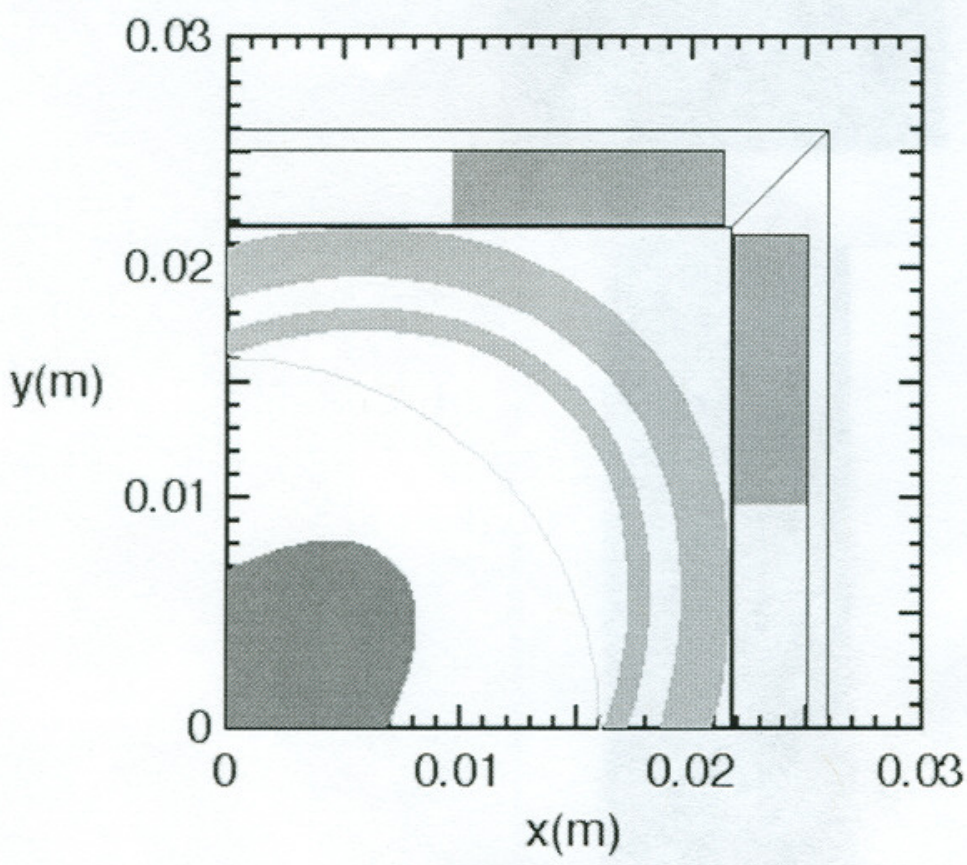

\section{$X-Y$ Projection}

Figure 8. A racetrack design using an elliptical vacuum pipe.

Although we may fabricate our first arrays with circular apertures, we would probably want to use elliptical apertures for a full-scale accelerator. In the following calculations, we assume elliptical apertures. Unfortunately, the beneficial effects of better mechanical design are offset by a more realistic treatment of emittance effects. In the following calculations, we obtain emittance as a function of beam current by scaling from the existing Berkeley injector[5]. Specifically, we assume Child-Langmuir emission. We also assume that the acceleration gap in the ion source scales linearly with the aperture of the source (constant optics) and that the voltage scales as the square root of the acceleration gap. Figure 9 gives average beam current density for the design with an elliptical vacuum pipe and including the effects of emmitance.

Recent progress in artificial-pinning-center conductors indicates that it may soon be possible to purchase niobium-titanium conductor that is significantly better than previous commercial conductors (approximately $5 \mathrm{kA} / \mathrm{mm}^{2}$ at $5 \mathrm{~T}$ compared to approximately $3 \mathrm{kA} / \mathrm{mm}^{2}$ for present conductor. Moreover, there has been steady progress toward higher current density in the copper portion of the superconducting strand. We assumed $1.5 \mathrm{kA} / \mathrm{mm}^{2}$ to obtain the results shown in Figure 9 but it may be possible to use $2.0 \mathrm{kA} / \mathrm{mm}^{2}$ in the future. The standard case shown in Figure 9 achieves an average 
beam current density of $2900 \mathrm{~A} / \mathrm{m}^{2}$ at a peak magnetic field of $3.4 \mathrm{~T}$. Use of the better superconductor gives $3100 \mathrm{~A} / \mathrm{m}^{2}$ and optimizes at a slightly higher magnetic field of 3.6 $\mathrm{T}$. The use of standard superconductor but for the copper gives $3150 \mathrm{~A} / \mathrm{m}^{2}$ at $3.6 \mathrm{~T}$. Finally the use of both the better superconductor and the higher current density in the copper gives $3390 \mathrm{~A} / \mathrm{m}^{2}$ at $4.1 \mathrm{~T}$.

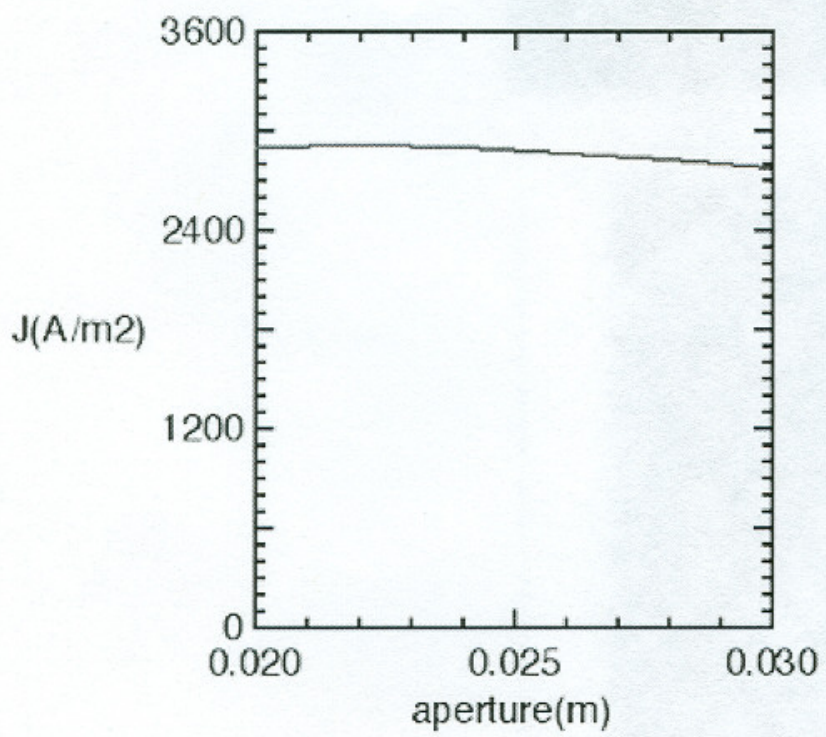

Figure 9. Average beam current as a function of aperture for a quadrupole with an elliptical beam pipe including effects of emittance.

As noted in Section 2.1, there are large differences in opinion concerning the proper choice of the misalignment parameter $\delta$. It is interesting to determine what would happen if one were able to align the beams well, say to $\delta=0.5 \mathrm{~mm}$ rather than $0.5 \mathrm{~cm}$. Figure 10 gives the results, together with the case shown in Figure 9. 


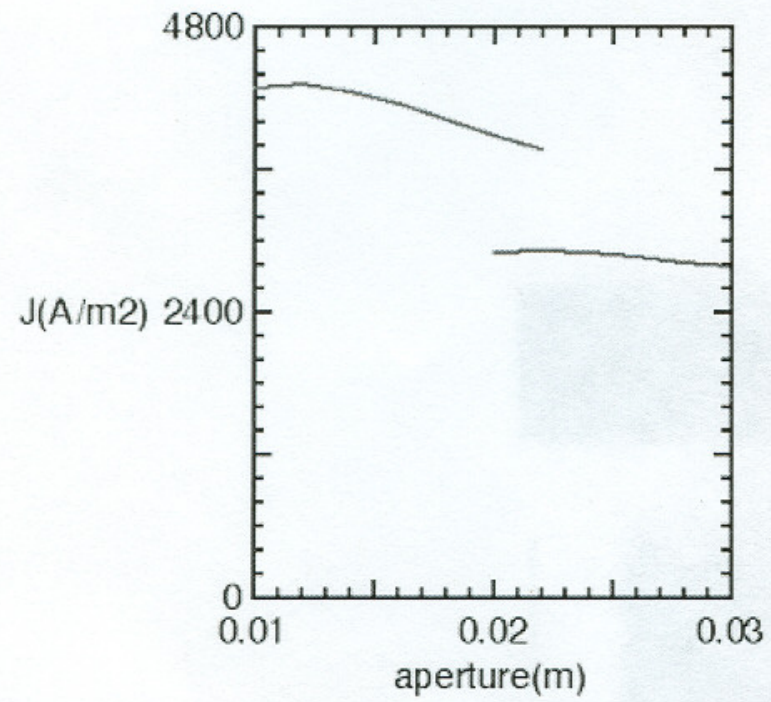

Figure 10. A comparison of two cases with different alignment parameters.

The red curve in figure 10 corresponds to $\delta=0.5 \mathrm{~mm}$ compared to the blue curve that corresponds to our standard case of $\delta=5.0 \mathrm{~mm}$. As expected, the optimal aperture is smaller in the case with small $\delta$. Also the average beam current density is larger, even at larger apertures and, for a given aperture, the current is larger. Thus, if one can improve alignment, one can reduce the size of the induction cores and improve the accelerator, either by reducing the aperture or by reducing the number of beams.

[1] J. D. Lawson, The Physics of Charged-Particle Beams, Clarendon Press, Oxford (1977).

[2] E. P. Lee, T. J. Fessenden, L. J. Laslett, IEEE Transactions on Nuclear Science, Vol. NS-32, No. 5, October 1985, p. 2489.

[3] L. Ahle, Lawrence Livermore National Laboratory, private communication.

[4 ] W. H. Press, S. A. Teukolsky, W. H. Vetterling, B. P. Flannery, Numerical Recipes in Fortran 77: The Art of Scientific Computing, Second Edition, Cambridge University Press, Cambridge (1992).

[5] F. Bieniosek, Lawrence Berkeley National Laboratory. private communication. The existing Berkeley injector has a normalized emmitance of approximately $1 \pi \cdot \mathrm{mr}$ at 1.8 $\mathrm{MeV}$ and $600 \mathrm{~mA}$. 


\section{Magnetic Fields in optimized HCX quadrupole (LLNL6r2)}

C. L. Goodzeit, M. J. Ball

\section{FOREWORD}

The configuration of the HCX optimized quadrupole as described in HCX-MAG-02-013101 has been analyzed using the 3D magneto-static field solver, AMPERES 6.0. The results include the field and gradient (for the case with and without the iron yoke) and the harmonic analysis of the field multipoles as a function of the axial length of the magnet (with the iron yoke).

\section{DESCRIPTION OF MODEL}

The dimension of the inner and outer coils and the position of the coils in the yoke are shown in Figures 1, 2 and 3.

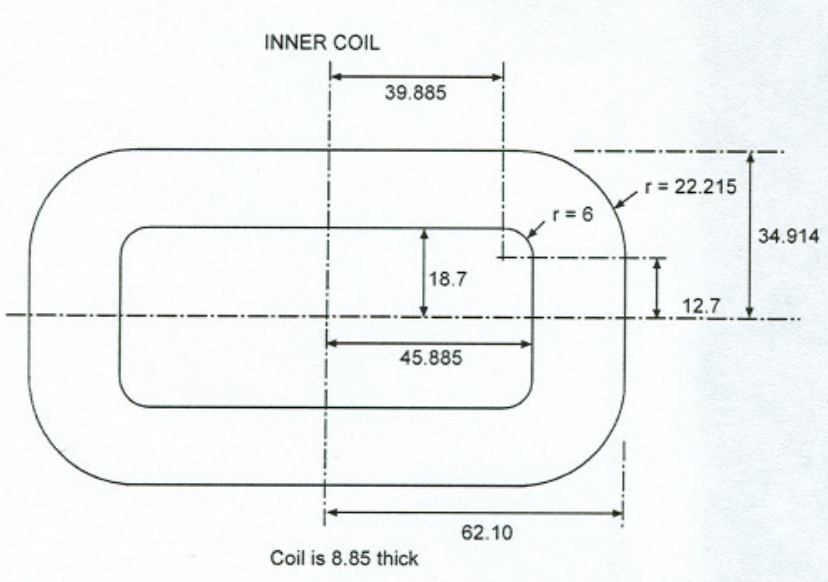

Figure 1

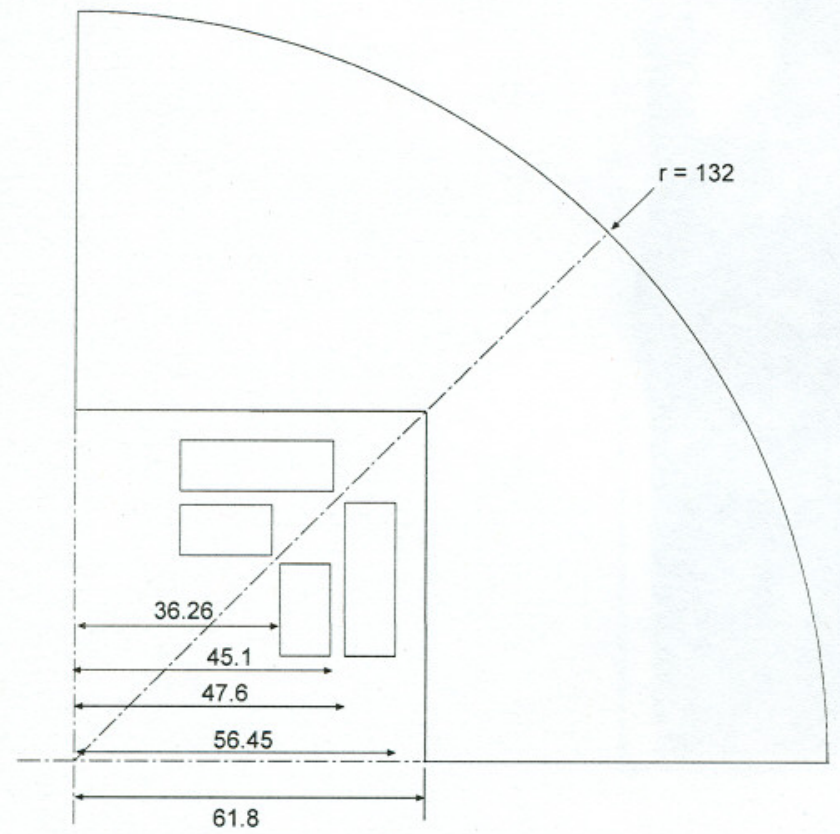

Ver. 1. HCX quad analysis

Page 1

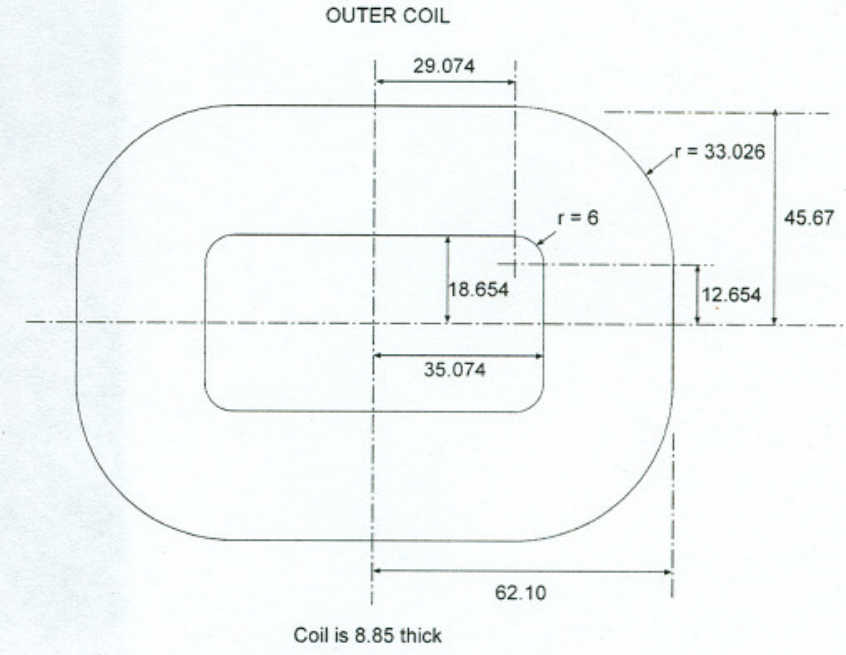

Figure 2

Figure 3

$10 / 7 / 02$ 
The three dimensional model as shown in Figure 4, has anti-symmetry about the $y=0$ plane and angular periodicity about the $y$-axis to form the complete magnet.

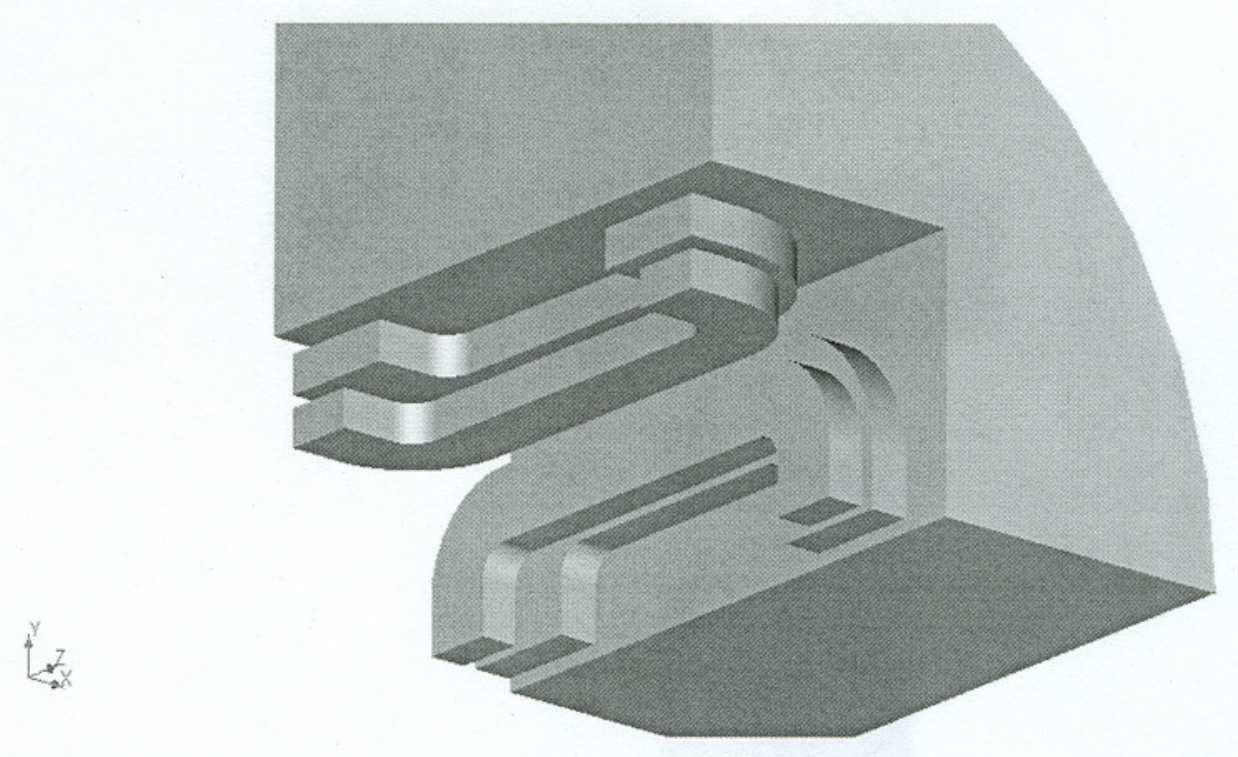

Figure 4

Other parameters associated with this model include a current density of $500 \mathrm{~A} / \mathrm{mm}^{2}$ applied to the coils and an iron yoke using M-45 iron. The saturation curve for the iron is shown in Figure 5 .

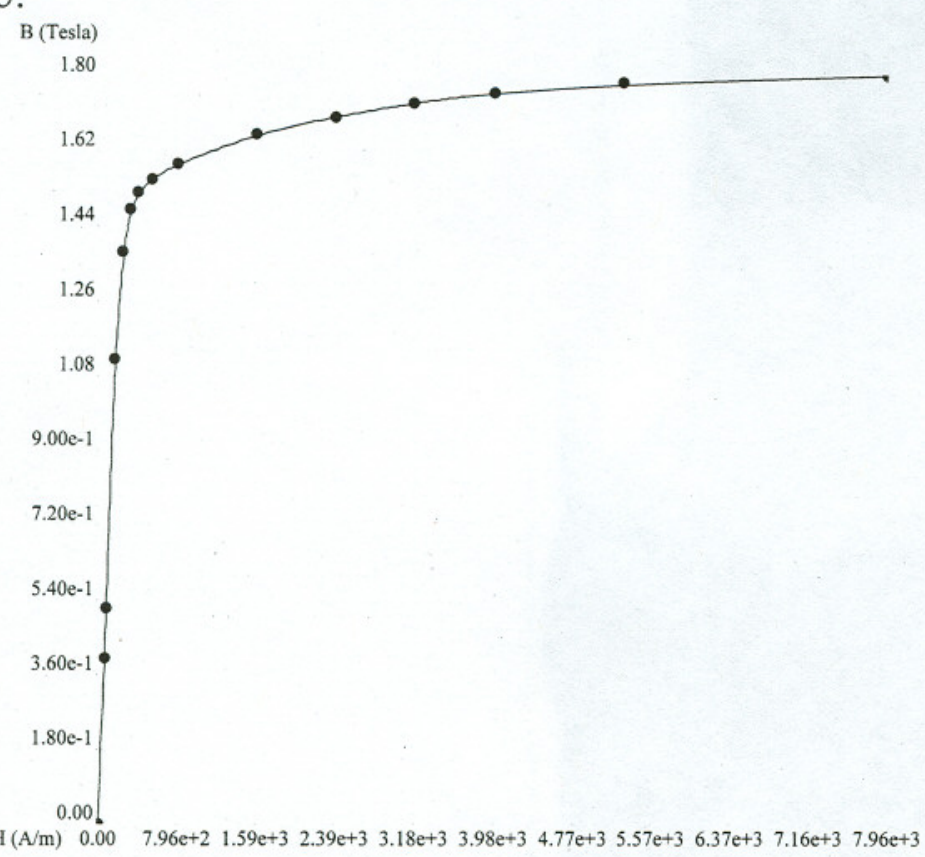

Figure 5. Saturation curve for M-45 steel. 


\section{Calculated Results from AMPERES}

3.1. Magnetic field strength and gradient

The magnetic field strength results are presented with and without the presence of the iron yoke. Figure 6 shows the value of the maximum radial field component at a reference radius of $25 \mathrm{~mm}$ along the length of the magnet. The coil ends are at $62.10 \mathrm{~mm}$.

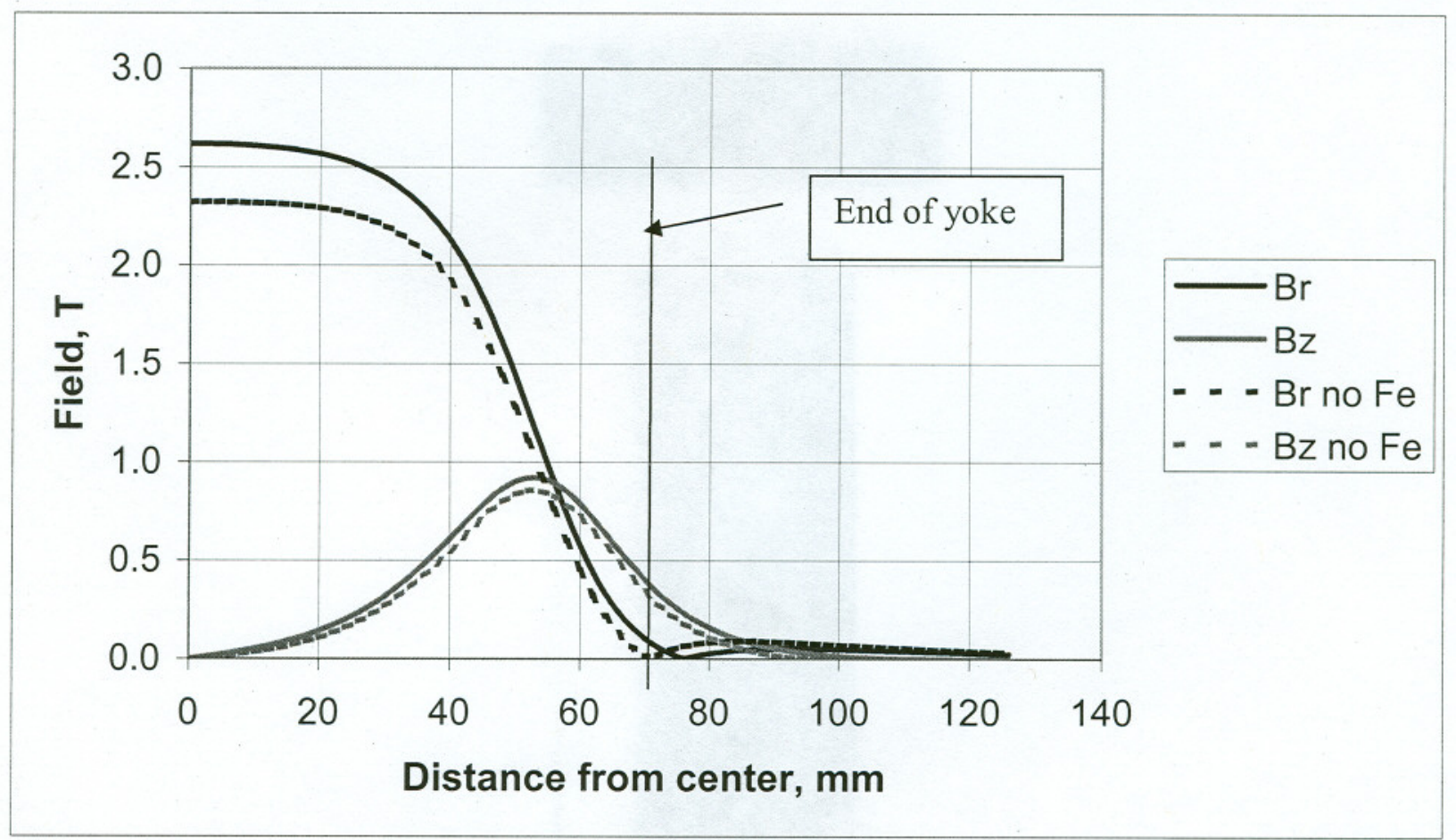

Figure 6. Maximum radial field component $(\mathrm{Br})$ and the solenoid field componet $(\mathrm{Bz})$ at a rerecence radius of $25 \mathrm{~mm}$ along the length of the magnet.

The maximum radial field at $\mathrm{r}=25 \mathrm{~mm}$ at the center of the magnet is $2.629 \mathrm{~T}$ with iron and $2.322 \mathrm{~T}$ without iron. It is noted that there is a significant solenoid field component, having a maximum value of $0.94 \mathrm{~T}$ (with $\mathrm{Fe}$ ) near the magnet end. The corresponding gradients, with and without iron, are shown in Figure 7. The maximum gradient at the magnet center is $105.15 \mathrm{~T} / \mathrm{m}$ with iron and $92.88 \mathrm{~T} / \mathrm{m}$ without it.

\section{HARMONIC ANALYSIS}

A multipole scan was made at $10 \mathrm{~mm}$ increments along the length of the coil. Only the allowed multipoles, the 12-pole and 20-pole, were present. The results are shown in Figure 8 in terms of multipole units, which are $10^{4}$ times the ratio of the multipole field to the fundamental field at a reference radius of $25 \mathrm{~mm}$. 
Appendix II, Part 1

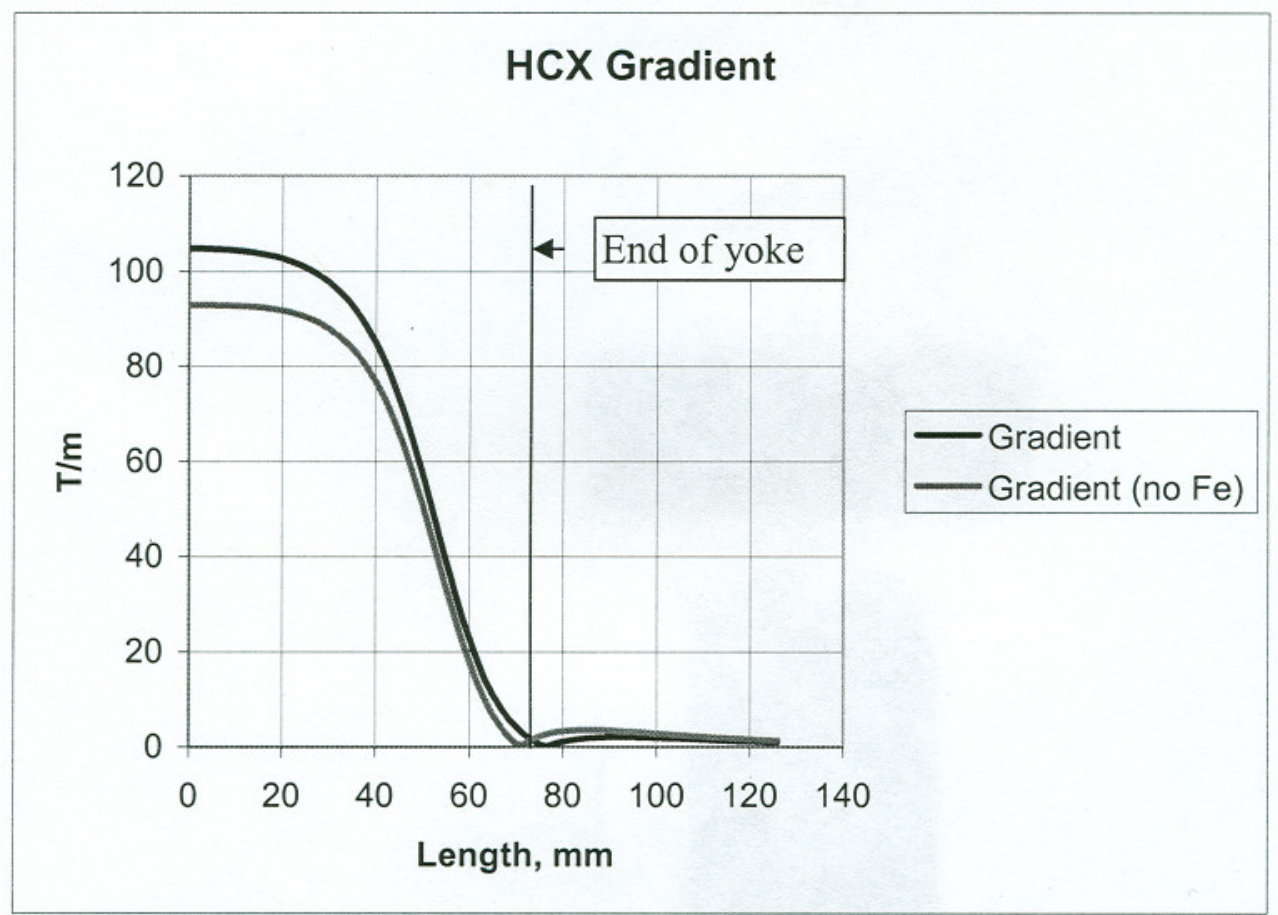

Figure 7. Gradient in HCX model with and without iron yoke.

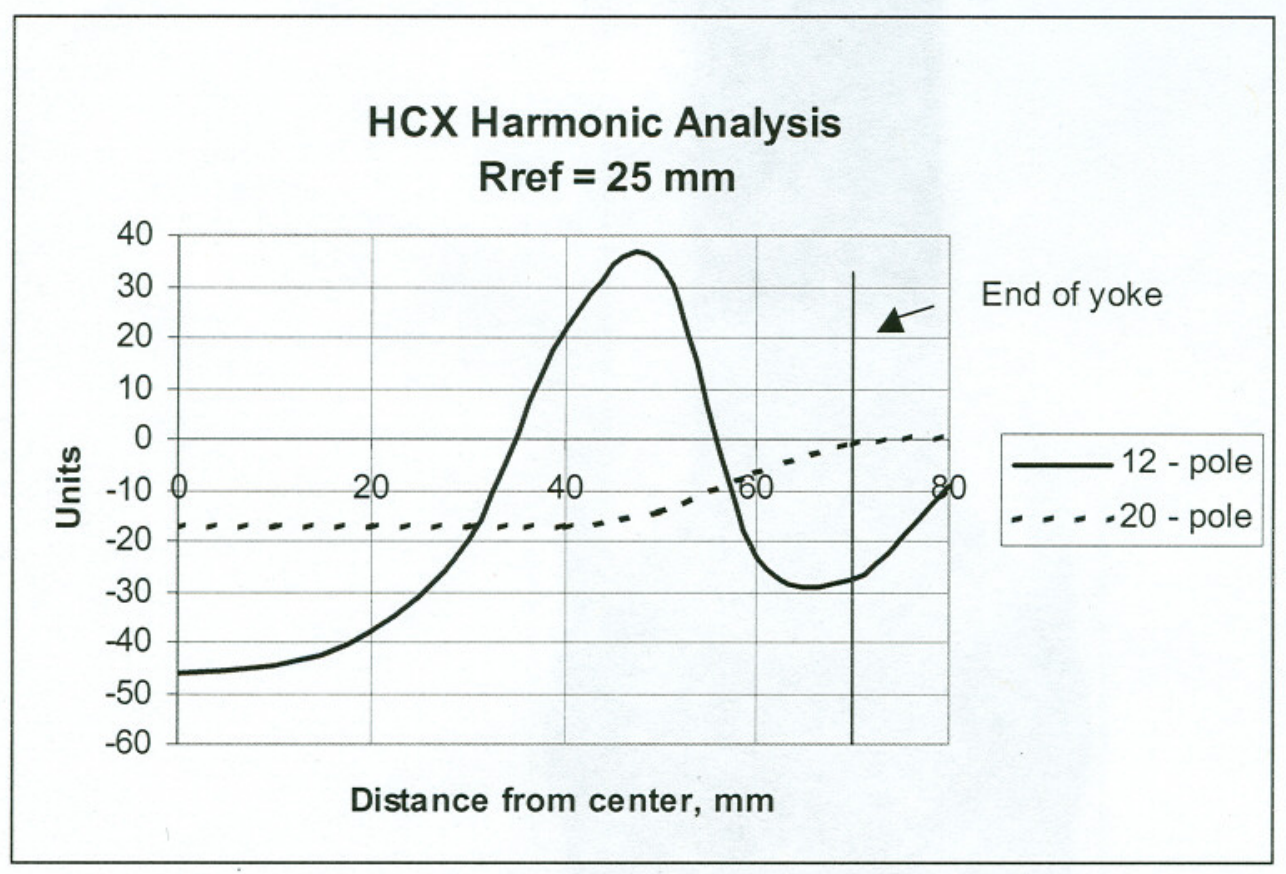

Figure 8. Multipole units along the length of HCX LLNL6r2 design. Iron yoke included. 
5. COMPARISON With PREVIOUS Results

A magnetic analysis of the LLNL6r2 design was reported in HCX-MAG-02-0131-01. The new AMPERES results are in qualitatively good agreement with those shown in Figure 6 of the previous report.

We show a comparison of this calculation with those previous results in the table below. In this table the comparison is made with the two sets of curves that were shown in Figure 6 of the previous report.

\begin{tabular}{|l|l|l|l|l|}
\hline & Units & This analysis & Previous (1) & Previous (2) \\
\hline Max radial field @ $r=25$ & $T$ & 2.629 & $n / a$ & \\
\hline Gradient at $z=0$ (with $\mathrm{Fe}$ ) & $\mathrm{T} / \mathrm{m}$ & 105.15 & $\sim 130$ & $\sim 105$ \\
\hline Gradient at $\mathrm{z}=0$ (no Fe) & $\mathrm{T} / \mathrm{m}$ & 92.88 & $\mathrm{n} / \mathrm{a}$ & \\
\hline 12 -pole @ $\mathrm{z}=0$ & Units & -46.14 & -5 & $\sim-40$ \\
\hline 20 - pole @ $\mathrm{z}=0$ & Units & -17.37 & $\sim-5$ & $\sim-5$ \\
\hline 12 - pole @ $\mathrm{z}=50$ & Units & 34.32 & 20 & 70 \\
\hline
\end{tabular}




\section{Forces in a Sample Array of HCX Inner Coils (LLNL6r2) \\ M. J. Ball, C. L. Goodzeit}

\section{FOREWORD}

A sample array of a nine cell pattern $(3 \times 3)$ of square quadrupoles using the inner coils of the LLNL6r2 design has been analyzed with the $3 \mathrm{D}$ magneto static analysis program AMPERES 6.0. The results for the relative field strength and the multipole content in each of the apertures in the array have been presented previously [1].

In this report we show the forces that appear in the array elements. The forces are tabulated as $\mathrm{x}, \mathrm{y}$, and $\mathrm{z}$ (axial) direction forces that act on each quadrant of a coil. The $\mathrm{x}-\mathrm{y}$ plane forces are used to determine the reaction forces that need to be applied to the external sides of the array coils to prevent outward deflection of the coil sides. It should be noted that the computed forces are for an array composed of coils with the dimensions that are shown in Figure 1. Thus, arrays made of longer coils will experience proportionally greater total $\mathrm{x}$ and $\mathrm{y}$ forces. The $\mathrm{z}$ direction (axial) forces indicate that the coils may require axial support.

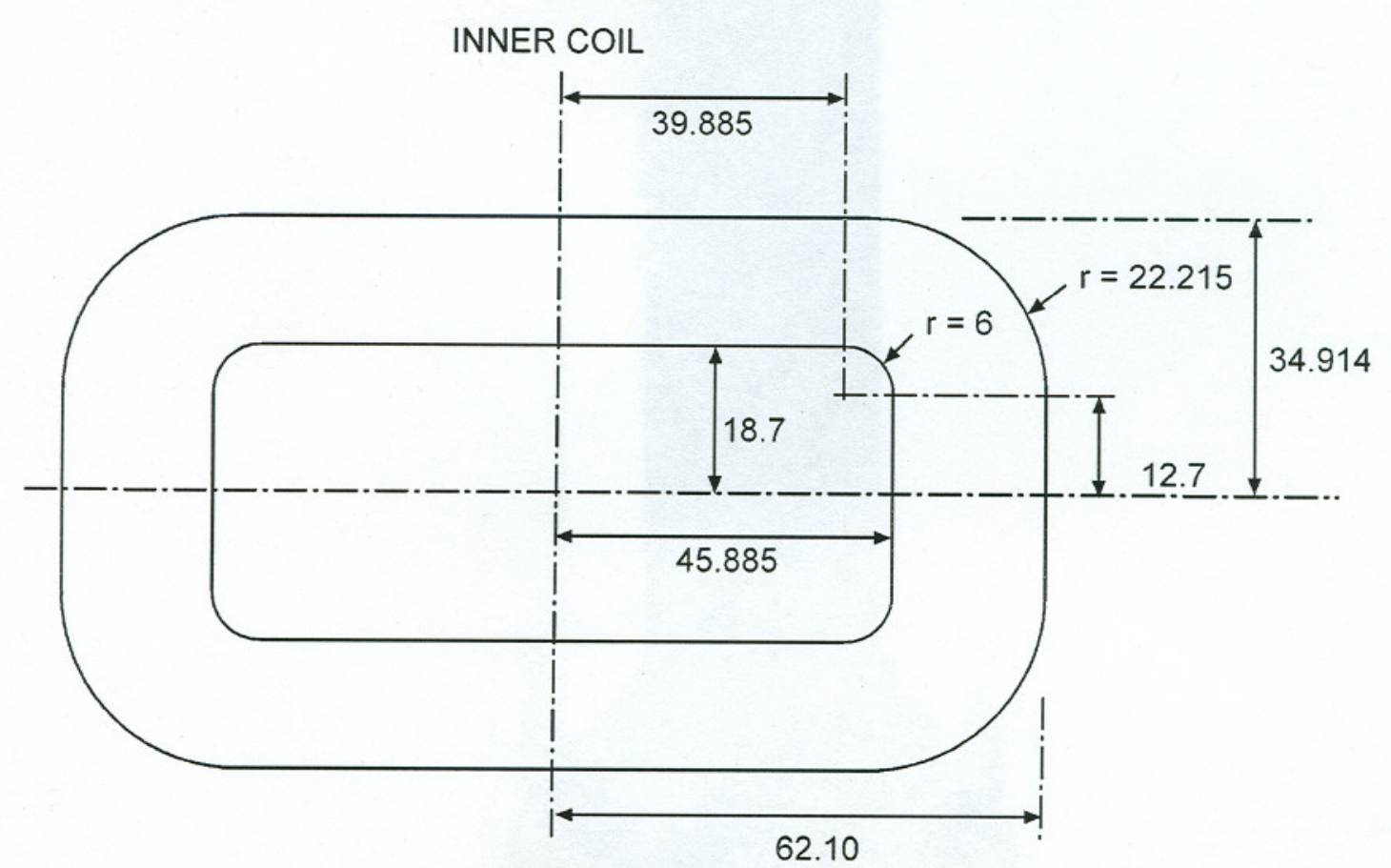

Coil is 8.85 thick

Figure 1. Side view of coil, with dimensions in $\mathrm{mm}$.

\section{DESCRIPTION OF MODEL}

An inner coil as shown in Figure 1 was used to form a 9 - cell, $(3 \times 3)$ array of square apertures. Using symmetry and periodicity, the array can be modeled from the upper right quadrant, as shown in Figure 2. It is seen that there are four types of cells in this model array. There is an interior cell, 1, a right-side cell, 2, a corner cell, 3, and a topside cell, 4 . 
Appendix II, Part 2

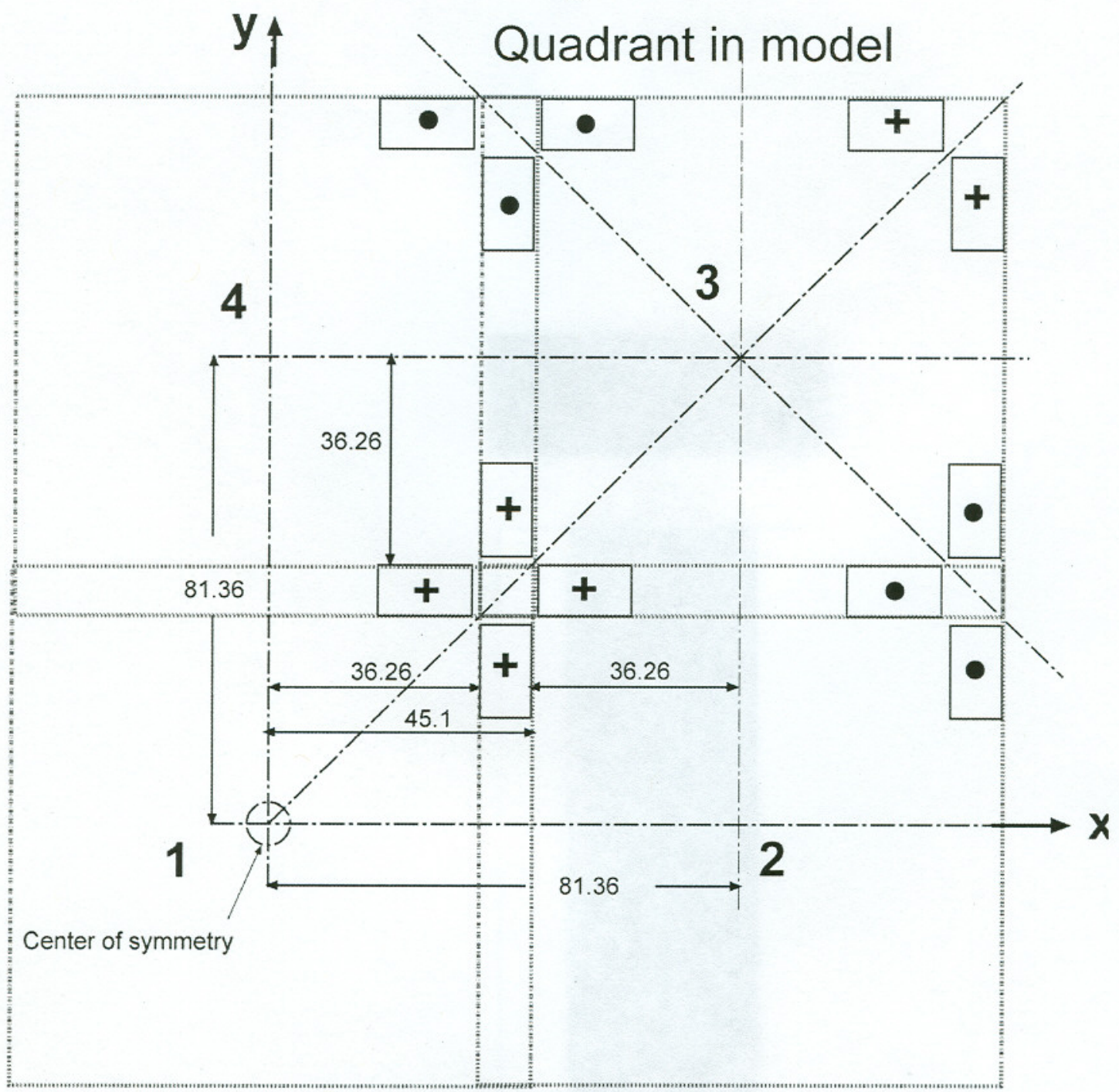

Figure 1. Array quadrant with current directions indicated by + (into plane) and · (out of plane).

\section{Calculated Results from AMPERES}

The magnetic field strength and forces on each of the coil sections in the array model were calculated with AMPERES ${ }^{\circledR}$ using a current density in the coils of $500 \mathrm{~A} / \mathrm{mm}^{2}$.

\subsection{Cell gradients}

The gradients in each cell are listed in Table 1.

Table 1. Gradient in cells at center $(\mathrm{z}=0)$ with $500 \mathrm{~A} / \mathrm{mm}^{2}$.

\begin{tabular}{|c|c|}
\hline \multicolumn{1}{|l|}{ Cell } & Gradient $(\mathrm{T} / \mathrm{m})$ \\
\hline 1 & 57.08 \\
\hline 2 & 56.21 \\
\hline 3 & 55.45 \\
\hline 4 & 56.21 \\
\hline
\end{tabular}




\subsection{Forces on coil quadrants.}

A qualitative understanding of the nature of the forces acting on the flat coils in the array can be obtained from a 2D analysis of the field produced by a single coil (not in an array) as shown in Figure 3. In this case the coil elements extend "infinitely" in the z-direction. With a current density of $500 \mathrm{~A} / \mathrm{mm}^{2}$, the maximum radial field component at the reference radius of 25 $\mathrm{mm}$ is $1.07 \mathrm{~T}(\mathrm{~g}=42.8 \mathrm{~T} / \mathrm{m})$.

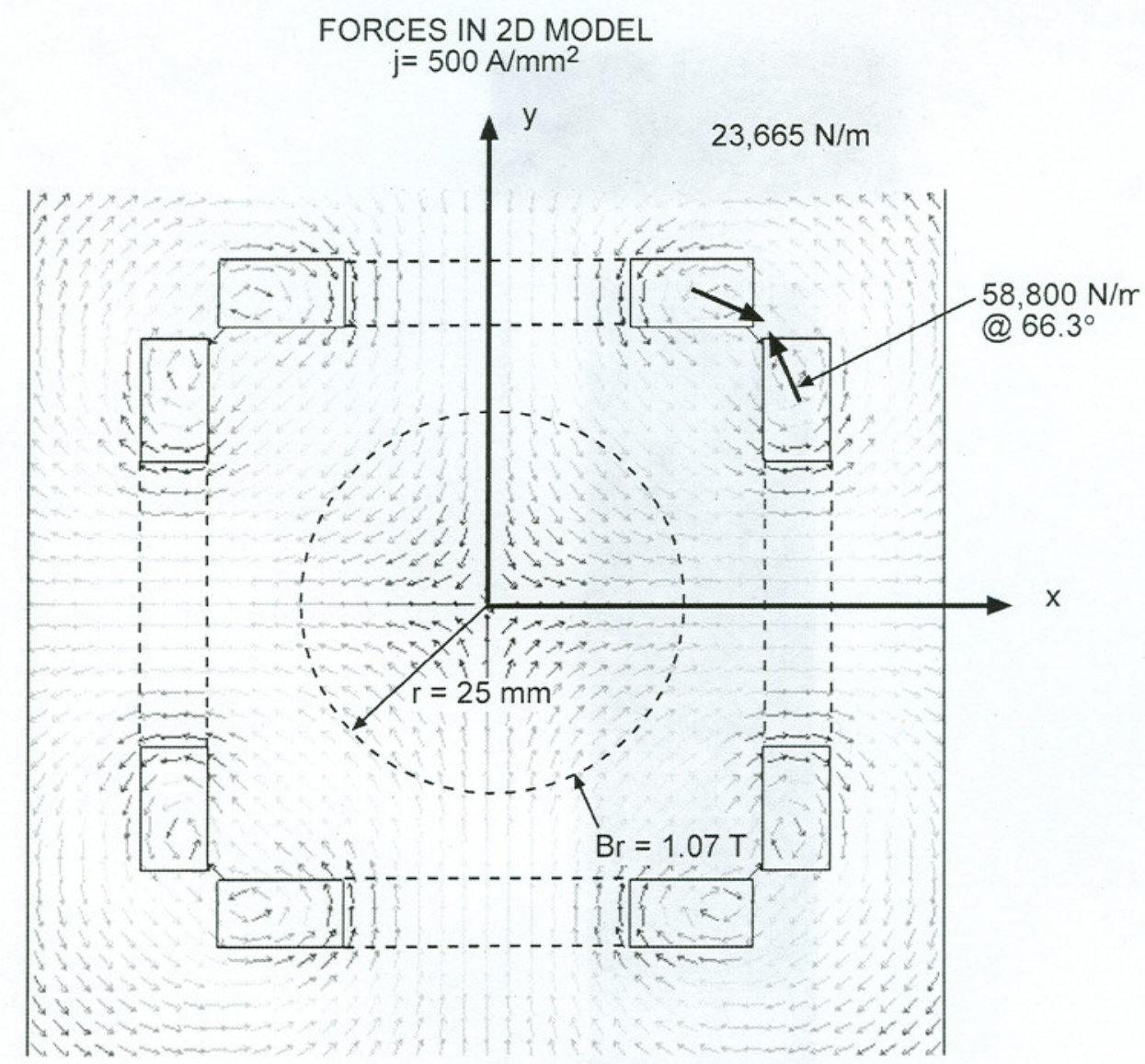

Figure 3. Field and forces on a single coil in a $2 \mathrm{D}$ model.

The directions of the forces in this 2D- model tend to make the coils become more circular (bow apart) in the $y-z$ plane and pull together in the $x-y$ plane. When the coils are placed in the array shown in Figure 4, similar forces act on each cell quadrant. (For the force calculations, each coil was considered as 3 sub-parts, as indicated by the numbers on coil C.)

Coils with unsupported faces $(\mathrm{D}, \mathrm{H}, \mathrm{J}$ and $\mathrm{I})$ require external reaction forces, indicated by the arrows $R$, to keep them in equilibrium. The values of $R_{1}$ and $R_{2}$, the reactions required to balance the internal forces generated in each of these coils, are listed in Table 3. For the interior coils that have opposing faces, the forces tending to make them circular can be (almost) balanced by placing rigid members between them, as indicated by the short red arrows in Figure 4 . Thus, the interior cells are in force equilibrium, with the $\mathrm{x}-\mathrm{y}$ forces on the coils balanced by the neighboring cells. 
Appendix II, Part 2

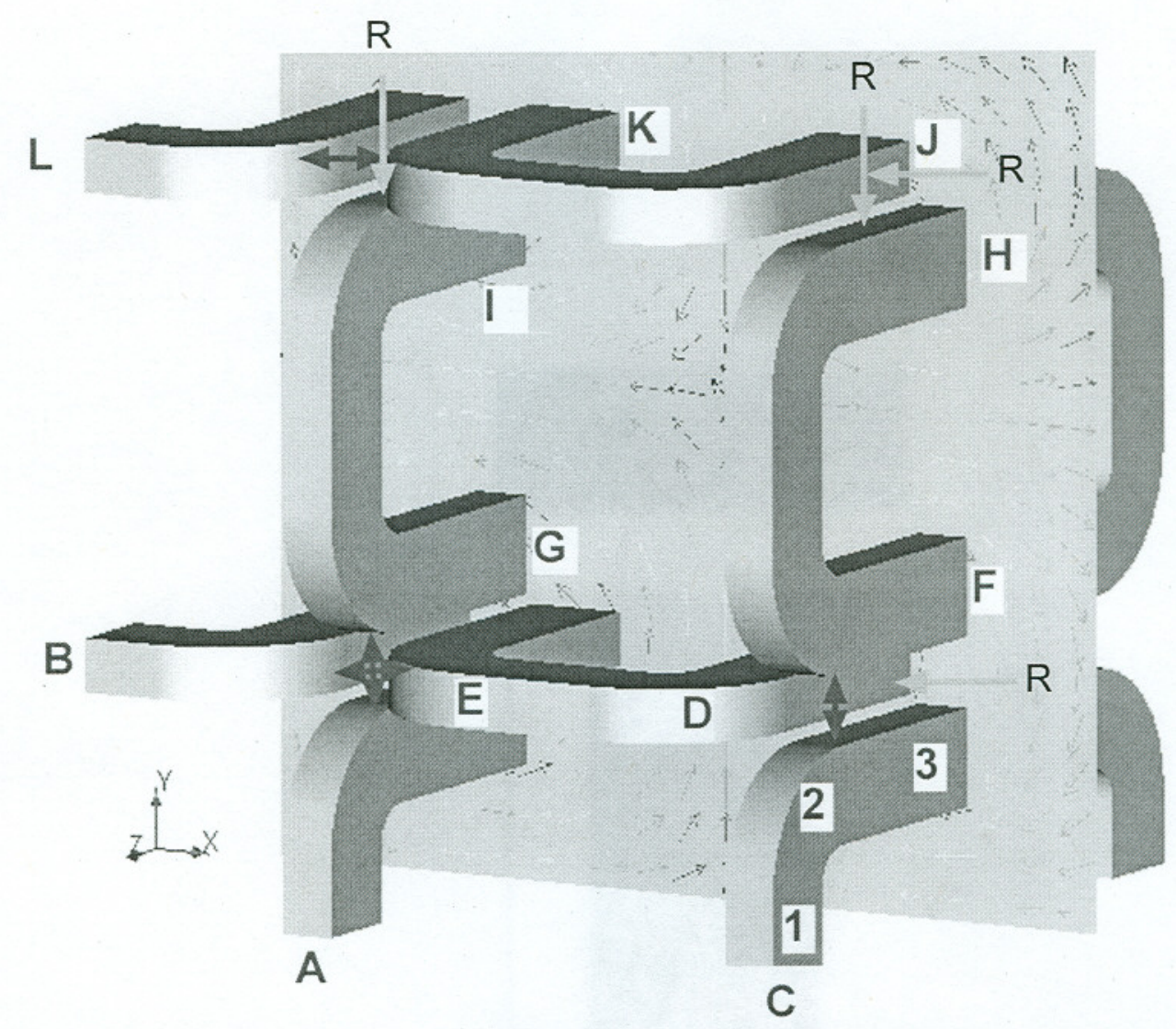

Figure 4. Diagram of coils in a $3 \times 3$ array model showing 12 labeled coil quadrants.

Table 2. Force components (N) acting on coil quadrants.

\begin{tabular}{|l|r|r|r|r|}
\hline $\begin{array}{l}\text { Total forces on } \\
\text { coils }\end{array}$ & \multicolumn{2}{|l|}{ Fx } & \multicolumn{2}{l|}{ Fy } \\
\hline Single coil & & 3132.8 & 1108.7 & 1364.0 \\
\hline A & & 41.7 & 5752.9 & 1854.0 \\
\hline B & & 5752.9 & 41.7 & 1854.0 \\
\hline C & & -1079.2 & 4202.0 & 1495.2 \\
\hline D & & 4776.5 & 59.6 & 1668.0 \\
\hline E & & -5647.7 & 40.3 & 1794.7 \\
\hline F & & -1069.5 & -4058.2 & 1418.2 \\
\hline G & & 40.3 & -5647.7 & 1794.7 \\
\hline H & & -1079.3 & 3236.4 & 1300.5 \\
\hline L & 59.6 & 4776.5 & 1668.0 \\
\hline J & & 3236.4 & -1079.3 & 1300.5 \\
\hline K & & -4058.2 & -1069.4 & 1418.2 \\
\hline L & & 4202.1 & -1079.1 & 1495.3 \\
\hline & & 5175.6 & 5175.7 & \\
\hline
\end{tabular}


Table 3. External reaction forces, $\mathrm{N} / \mathrm{mm}^{2}$ necessary to keep cells in equilibrium.

\begin{tabular}{|c|c|c|}
\hline Coils & $\mathrm{R}_{1}$ & $\mathrm{R}_{2}$ \\
\hline $\mathrm{D}, \mathrm{I}$ & 97.6 & \\
\hline $\mathrm{H}, \mathrm{J}$ & & 66.2 \\
\hline
\end{tabular}

\subsection{End Forces}

The end forces ( $\mathrm{z}$-direction forces) on each of the coil sections $\mathrm{A}-\mathrm{L}$ have an average value of $1570 \mathrm{~N}$. When applied to the coil cross-section, this produces a tensile stress of $10.9 \mathrm{~N} / \mathrm{mm}^{2}$ (1750 psi). This stress may be low enough to consider that the coils are self-supporting against the end forces. However, to determine if this is feasible, the bending effect at the end will be considered in a separate report on the structural analysis.

\section{Summary of Results}

We have computed the 3D force components acting on the elements of a $3 \times 3$ array composed of inner coils of the LLNL6r2 design at a current density of $500 \mathrm{~A} / \mathrm{mm}^{2}$. This analysis shows that the central elements in the array are in force equilibrium with their neighbors; this would prevent outward deflection (bowing) of the coils. However, the outer array elements require external support to maintain force equilibrium and hence prevent outward coil deflections. The end forces may be at a low enough level to consider that the coils may be selfsupporting in that direction. However, this computation of the forces provides the necessary input for a subsequent structural analysis on both interior and exterior cell coils in order to determine the necessary reinforcement required for each case.

1 C. Goodzeit, M. Ball, "Magnetic Fields in a Double-Helix Quadruple 3 x 3 Array", 10/07/02. 


\title{
Magnetic Fields in Example Array Magnets for SBIR Phase II Proposal
}

\author{
C. L. Goodzeit, M. J. Ball
}

\section{FOREWORD}

We present a magnetic analysis of two examples of quadrupole magnets that could be used in array experiments for a Phase II SBIR. One is based on the LLNL6r2 (flat coil) design. The second is a double-helix quadrupole design [1]. It is noted here that the parameters for these magnets will be finalized in Phase II depending on the actual configuration selected for the magnets used in sample arrays. However, for the purpose of this analysis, both designs have a coil aperture of $48 \mathrm{~mm}$ and are $320 \mathrm{~mm}$ in physical length. They produce a gradient of $\sim 110$ $\mathrm{T} / \mathrm{m}$ including a field enhancement of $26 \%$ [2] from neighboring magnets if placed in an array.

\section{DESCRIPTION OF MAGNETS}

\subsection{Flat coil design}

The flat coil magnet consists of a double pancake coil embedded in an aluminum alloy frame as shown in Figure 1. Each coil is wound with 12 turns of 24-strand flat cable made from $0.37 \mathrm{~mm} \mathrm{NbTi}$ strand with 1.8:1 Cu:SC. The cable is flattened so that the compaction is about $90 \%$ of the nominal thickness. The cable is insulated by a $50 \%$ overlap wrap of 1 -mil Kapton.

24 strand ( $0.37 \mathrm{dia}$.) bare cable dimensions
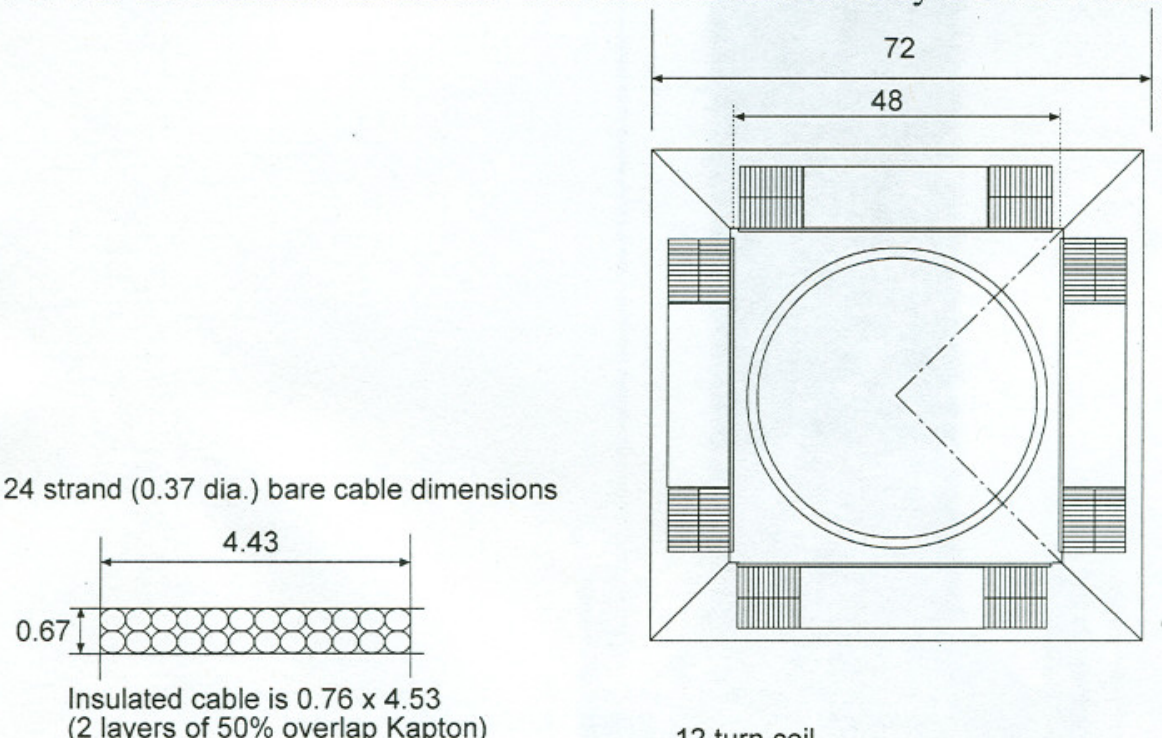

Insulated cable is $0.76 \times 4.53$

2 layers of $50 \%$ overlap Kapton)

\section{2 turn coil}

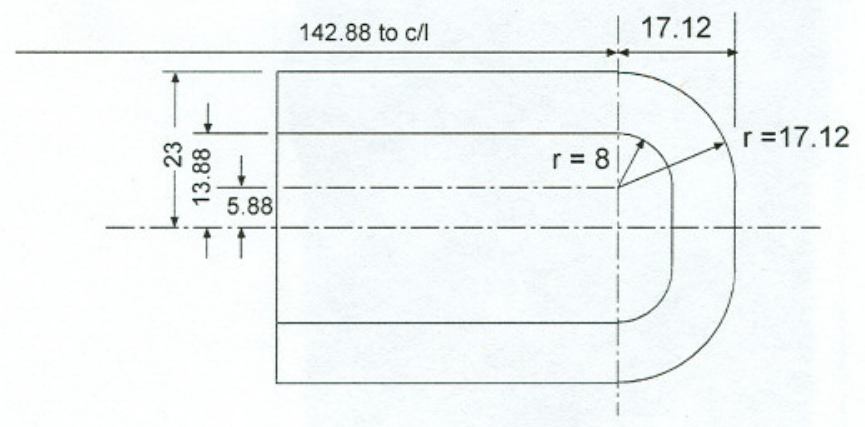

Figure 1. Example flat coil array element. Suggested use: central element in an edge termination test. 


\subsection{Double-helix quadrupole}

The double-helix quadrupole which has been described in [1] is a novel magnet design that produces a quadrupole field by modulating the axial position of the conductor path in a helically wound solenoid with a periodicity of 2 . Figure 2 shows the conductor arrangement for a pair of double-helix quadrupole coils.

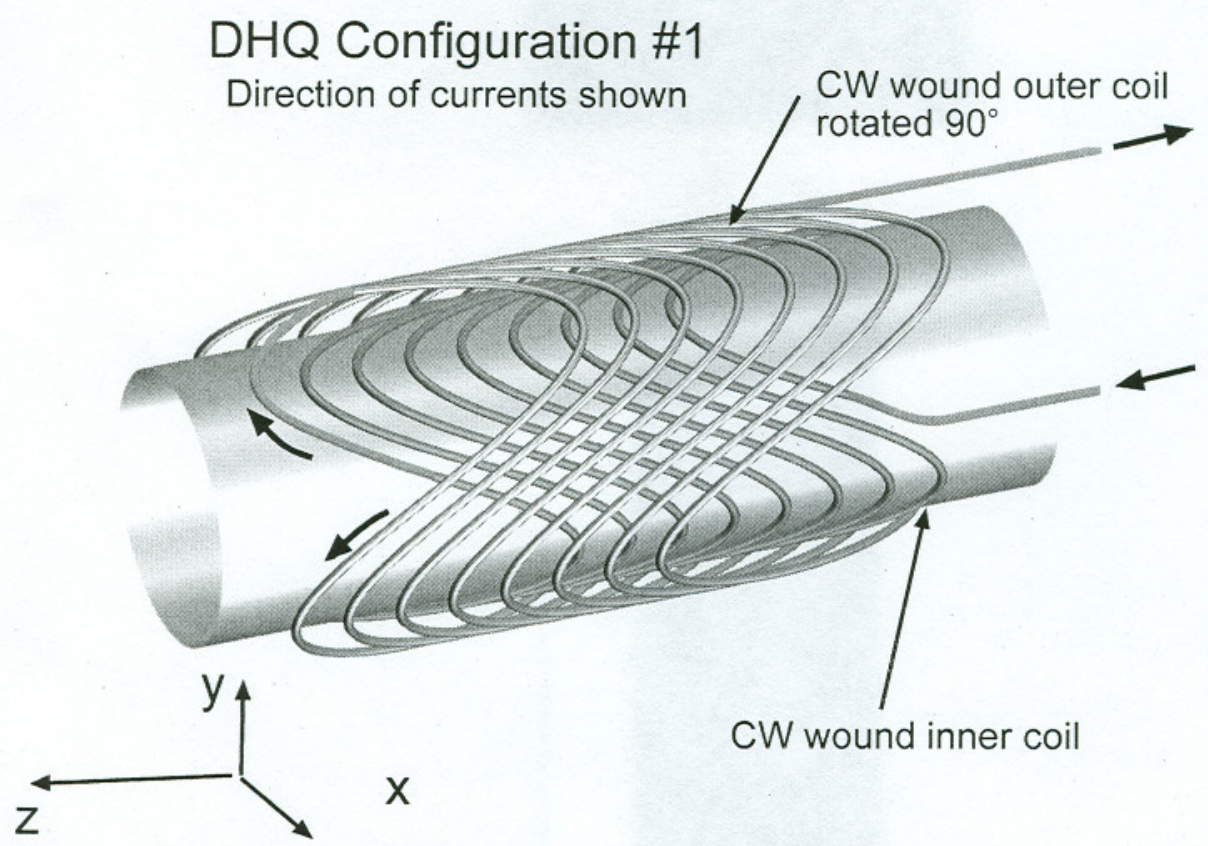

Figure 2. Arrangement of conductors in a 2-layer double-helix quadrupole.

It is necessary to use pairs of these windings in order to cancel the solenoid field in the aperture of the magnet and at the same time add the quadrupole field from each layer. The magnet used in this analysis has a 4-layer double-helix quadrupole coil mounted on a bore tube with I.D. of $41 \mathrm{~mm}$. Such magnets mounted in a $3 \times 3$ array in a structural aluminum alloy frame are shown in Figure 3.

This design uses a 37-strand NbTi cable of the same conductor as used in the flat coil model example. However, this is a round conductor with a bare diameter of $2.6 \mathrm{~mm}$. Insulation is not required since the cable is supported in grooves with a $1 \mathrm{~mm}$ web and thus the effective conductor width is $3.6 \mathrm{~mm}$ in this example. 


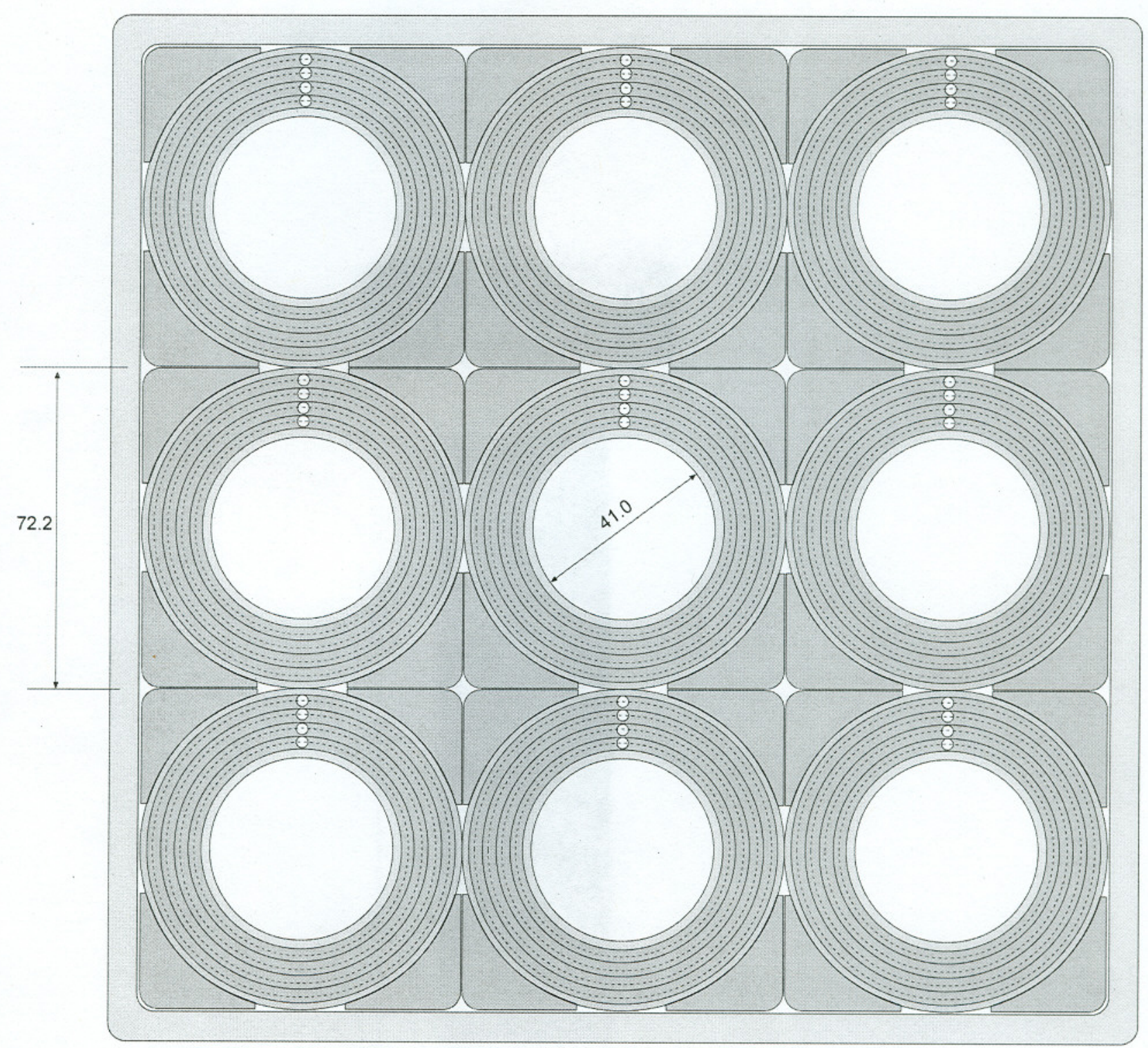

Figure 3. Double-helix quadrupoles mounted in a $3 \times 3$ array. Composite segments are bonded to the outer composite tube of the coil to form a precise square cross section.

\section{Magnet data And PARAMETERS}

\subsection{Superconductor}

The properties of the superconducting strand and cable used in each magnet type are listed in Table 1. 
Table 1. Strand and Cable Properties

\begin{tabular}{|l|r|r|c|}
\cline { 2 - 4 } \multicolumn{1}{c|}{} & Double-helix & Flat Coil & Units \\
\hline Cable style & Round & Flat & \\
\hline No. of strands of NbTi composite & 37 & 24 & \\
\hline Strand dia. & 0.37 & 0.37 & $\mathrm{~mm}$ \\
\hline Cable, dia., (bare) & 2.58 & $\mathrm{n} / \mathrm{a}$ & $\mathrm{mm}$ \\
\hline Cable width (bare) & $\mathrm{n} / \mathrm{a}$ & 4.43 & $\mathrm{~mm}$ \\
\hline Cable thickness (bare) & $\mathrm{n} / \mathrm{a}$ & 0.67 & $\mathrm{~mm}$ \\
\hline Cu/NbTi & 1.8 & 1.8 & \\
\hline Cu area in cable & 2.54 & 1.65 & $\mathrm{~mm}^{2}$ \\
\hline NbTi area in cable & 1.41 & 0.92 & $\mathrm{~mm}^{2}$ \\
\hline Degradation in cabling & $0.00 \%$ & $5.00 \%$ & \\
\hline
\end{tabular}

\subsection{Superconductor use comparison}

The amount of superconductor used in each of the magnets per meter of the two dimensional cross-section is computed from the coil geometry and cable properties. These parameters are listed in Table 2.

Table 2. Amount of superconductor used in both design types.

\begin{tabular}{|c|c|c|c|c|c|c|c|}
\hline & \multicolumn{5}{|c|}{ Double-helix } & \multirow[t]{2}{*}{ Flat Coil } & \multirow[t]{2}{*}{ Units } \\
\hline & Coil1 & Coil2 & Coil3 & Coil4 & Total & & \\
\hline Mean radius & 24.00 & 27.58 & 31.17 & 34.75 & & & $\mathrm{~mm}$ \\
\hline Arc length/turn & 256.35 & 294.62 & 332.89 & 371.16 & & 1000 & $\mathrm{~mm}$ \\
\hline Turns/m & 125 & 125 & 125 & 125 & & 192 & \\
\hline Meters of cable/m & 32.00 & 36.77 & 41.55 & 46.33 & 156.65 & 192 & $\mathrm{~m}$ \\
\hline $\mathrm{kg}$ of cable/m & 1.03 & 1.18 & 1.34 & 1.49 & 5.04 & 4.77 & $\mathrm{~kg}$ \\
\hline
\end{tabular}

In the magnets, as configured, the DHQ design uses 5.6\% more superconductor than the flat coil design; however, this tends to be offset by the $5 \%$ degradation applied to the flat cable and that the flat cable is more costly to produce than the round cable. Thus, we cannot conclude without further study that the superconductor cost would be more costly in one design or the other.

3.3. Operating parameters of the magnet designs.

Each of these magnets is designed to have a maximum field on the superconductor of $\sim 3 \mathrm{~T}$ assuming that there is a $26 \%$ field enhancement due to being in an array. The operating parameters are listed in Table 3.

Table 3. Operating parameters of magnets with $26 \%$ array enhancement.

\begin{tabular}{|l|r|r|c|}
\cline { 2 - 4 } \multicolumn{1}{c|}{} & \multicolumn{1}{c|}{ Double-helix } & Flat Coil & Units \\
\hline Operating current & 4302 & 2788 & $\mathrm{~A}$ \\
\hline Operating temp & 4.35 & 4.35 & $\mathrm{~K}$ \\
\hline $\mathrm{J} \mathrm{Cu}$ & 1691 & 1690 & $\mathrm{~A} / \mathrm{mm}^{2}$ \\
\hline $\mathrm{J} \mathrm{NbTi}$ & 3044 & 3042 & $\mathrm{~A} / \mathrm{mm}^{2}$ \\
\hline Max field, T & 2.96 & 2.96 & $\mathrm{~T}$ \\
\hline $\mathrm{JC}$, strand, 6T, 4.23K & 2252 & 2252 & $\mathrm{~A} / \mathrm{mm}^{2}$ \\
\hline
\end{tabular}




\begin{tabular}{|l|r|r|c|}
\hline JC, strand, operating & 4056 & 3853 & $\mathrm{~A} / \mathrm{mm}^{2}$ \\
\hline Margin (JC) & $24.9 \%$ & $21.1 \%$ & \\
\hline Margin (Temperature) & 1.01 & 0.86 & $\mathrm{~K}$ \\
\hline Inductance & 1.2 & 2.9 & $\mathrm{mH}$ \\
\hline
\end{tabular}

\section{MaGNET FIELD ANALYSIS OF THE TWO DESIGNS}

The magnetic field in the magnets was calculated with the magneto static solver AMPERES 6.0. The results are presented here for a single magnet not included in an array and thus there is no field enhancement included. The flat coil magnet had a physical length of $320 \mathrm{~mm}$. The modulation factor used for this example of the DHQ was $\mathrm{k}=1$ (see [1]); thus a single turn of the outer coil occupies an axial distance of $75.3 \mathrm{~mm}$. In this case 31 turns were used with helical adavance $8 \mathrm{~mm}$ per turn to achieve a total physical length of $323 \mathrm{~mm}$ for the DHQ used in this example. The reference radius used for the output of the magnetic field components was $16 \mathrm{~mm}$.

\subsection{Magnetic field strength (gradient)}

The field gradient along the length of the magnets from the centerline is plotted is in Figure 4 for the DHQ, the flat coil design and the flat coil design with an iron pole piece.

\section{Gradient in DHQ and Flat Coil Magnets}

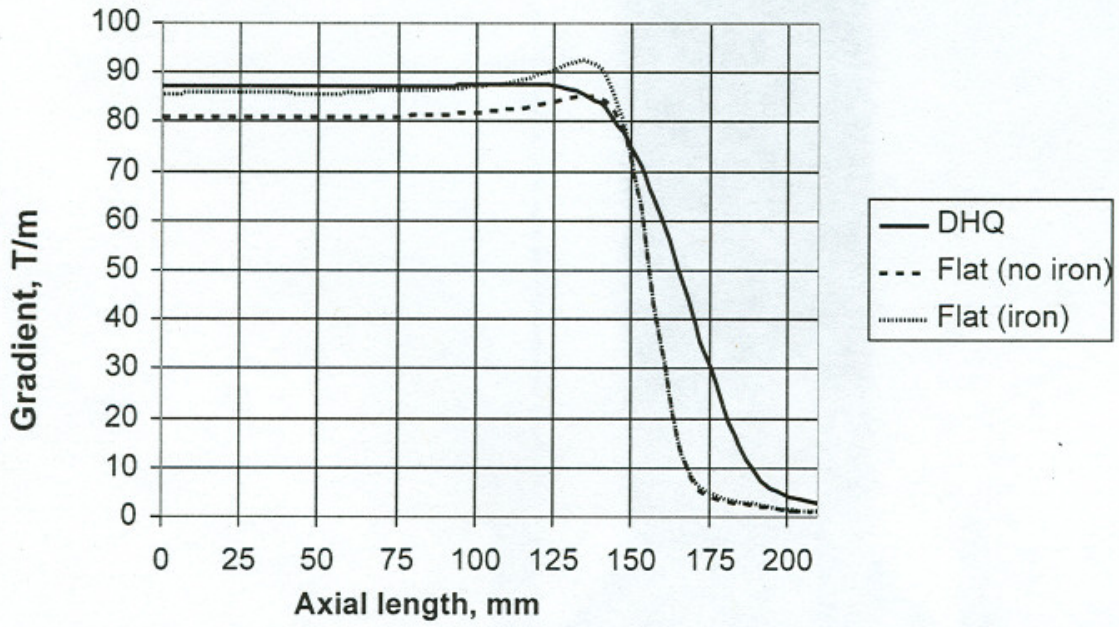

Figure 4. Gradients in the DHQ and the Flat Coil Design.

Although the field from the flat coil designs drops more abruptly at the end, the DHQ characteristically produced a more uniform field along its length without the end enhancement effect seen in the flat coil.

\subsection{Harmonic Analysis}

\subsubsection{Harmonic content at magnet center}

The principal multipoles at the center of each magnet are listed in Table 4 with values in gauss shown at the reference radius of $16 \mathrm{~mm}$. As expected the DHQ shows virtually zero multipoles that are allowed by quadrupole symmetry. No attempt was made to optimize the allowed multipoles in the flat coils and thus there are significant allowed components. The components that are not due to quadrupole symmetry are also listed. They are the dipole for the DHQ and the octupole with the use of the iron pole piece in the flat coil design. 


